Sharif University of Technology
Scientia Iranica
SCIENTIA
I RAN ICA
http://scientiairanica.sharif.edu

\title{
Novel technique for dynamic analysis of shear frames based on energy balance equations
}

\author{
M. Jalili Sadr Abad $^{a}$, M. Mahmoudi ${ }^{a, *}$, and E. Dowell ${ }^{b}$ \\ a. Department of Civil Engineering, Shahid Rajaee Teacher Training University, Tehran, P.O. Box 16788-15844, Iran. \\ b. Department of Mechanical Engineering and Materials Science, Duke University, Durham, USA. \\ Received 4 October 2017; received in revised form 7 April 2018; accepted 28 July 2018
}

\author{
KEYWORDS \\ Numerical technique; \\ Dynamic analysis; \\ Shear-frames; \\ Energy balance \\ equations; \\ Coupled equations; \\ Elimination of \\ discontinuous \\ velocities.
}

\begin{abstract}
In this paper, an efficient computational solution technique based on the energy balance equations is presented to perform the dynamic analysis of shear frames, as an example of a multi-degree-of-freedom system. After deriving the dynamic energy balance equations for these systems, a new mathematical solution technique called elimination of discontinuous velocities is proposed to solve a set of coupled quadratic algebraic equations. The method will be illustrated for the free vibration of a two-story structure. Subsequently, the damped dynamic response of a three-story shear frame, which is subjected to harmonic loading, is considered. Finally, the analysis of a three-story shear building under horizontal earthquake load, as one of the most common problems in earthquake engineering, is studied. The results show that this method has acceptable and greater accuracy than other techniques; it is faster than modal analysis and does not require adjusting and calibrating the stability parameter as compared to a time integration method like the Newmark method.

(C) 2020 Sharif University of Technology. All rights reserved.
\end{abstract}

\section{Introduction}

Generally, in all engineering fields that deal with structural design, understanding the dynamic behavior of structures is very important [1]. In this context, although the applications of structural dynamics in aerospace engineering, civil engineering, engineering mechanics, and mechanical engineering are different, the principles and solution techniques are basically the same [2]. Accordingly, dynamic analysis plays a vital role in analyzing the dynamic response of buildings [3], dams [4,5], and bridges [6] to earthquakes. Control of very tall and slender buildings is among the most

\footnotetext{
*. Corresponding author. Tel.: +982122970060;

Fax: + 982122970033

E-mail addresses: m.jalili@srttu.edu (M. Jalili Sadr Abad);

m.mahmoudi@srttu.edu (M. Mahmoudi);

earl.dowell@duke.edu (E.Dowell)
}

doi: $10.24200 /$ sci. 2018.20790 important issues for civil engineering researchers and has frequently been investigated in recent years.

Although almost all practical structures are Multiple-Degree-of-Freedom (MDF) systems because of the distribution of dynamic properties such as mass in real systems, so many DOFs are required to determine the vibrational motion [7]. In addition, as is known, a greater number of DOFs will increase the complexity of solving a vibration problem. Thus, in engineering applications, we prefer to work with fewer DOFs without losing too much accuracy. For example, in the modeling of dynamical systems, when much of the mass of the system is concentrated in the structure area, simple structures (such as water tank) can be idealized as a system with a lumped mass (SDF SingleDegree-of-Freedom (SDF) systems) [8]. Moreover, under some conditions such as when a mathematical function can express the variation of the mass and stiffness of the structure, the real system is considered as a generalized SDF system [3]. Furthermore, there 
are other techniques for reducing the dynamical DOFs of large-order systems under some conditions, e.g., refer to [9-11]. However, in many cases, in practical engineering works, there lies no possibility of simplifying a real system to an SDF system; therefore, an MDF dynamic analysis needs to be conducted.

There are various methods for evaluating the dynamic response of MDF systems. For example, in some particular cases, by applying the mathematical tools such as Fourier and Laplace integral transforms, an exact solution to these problems can be obtained $[12,13]$. Moreover, modal analysis is a conventional approach to evaluating the response of MDF structures, which are subjected to dynamic loads. One of the disadvantages of this method is its limitation for structures with nonlinear behavior [1]. However, some researchers have tried to modify the modal analysis in order to use it for nonlinear analyses; however, there is no comprehensive method yet for performing the modal analysis of nonlinear structures (see, for example, [14-20]). Even though there exist some techniques to determine the eigenvalues and eigenvectors of large-order systems (e.g., refer to [21,22]); as DOFs increase, the calculation of eigenvalues and eigenvectors is particularly difficult, which is another disadvantage of this approach.

In engineering analyses, the most general solution method for performing dynamic analysis is an incremental method or a step-by-step direct time integration technique in which the equilibrium equations are solved at times $\Delta t, 2 \Delta t, 3 \Delta t$, etc. [1]. In this category, Newmark [23], Houbolt [24], and Wilson et al. [25] are some common implicit methods, and central difference method is one of the well-known explicit methods [26]. Stability and accuracy of these methods are essential in the practical analysis [27-31]; therefore, it is very important to use accurate and numerically efficient techniques in computer programs [32]. As a result of the large computational requirements, it can take a significant amount of time to solve structural systems with just a few hundred DOFs [26]. In addition, artificial or numerical damping must be added to most incremental solution methods to obtain stable solutions. For this reason, engineers must be very careful with the interpretation of the results [1]. Here, it should be noted that the artificial damping, which is defined as the reduction of the displacement amplitude with time for an undamped system [33], is different from the damping property of the structures.

Applying energy balance equations, proposed in this study, can be an alternative approach to evaluating the dynamic response of a multi-dimensional system. In this context, for instance, the energy conservation and dissipation properties of time-integration methods were investigated by Acary [34] for the non-smooth elastodynamics with contact. Even though several researchers in various fields such as hydrodynamic [35], aerospace [36,37], and CFD (Computational Fluid Dynamics) $[38,39]$ have studied the energy method to determine the response of their dynamic systems, yet insignificant attention has been paid to this topic in structural dynamics, except for a few studies that have often tried to use Hamilton's Principle in order to calculate the frequency of simple SDF structures (see, for example $[40,41])$.

Accordingly, this study aims to present a new numerical step-by-step method based on the energy equations for MDF shear frames. The main idea of this approach was introduced first in [42] for linear and nonlinear SDF systems, and, in the present paper, this technique is intended to be generalized to linear MDF structures. This method in the absence of damping leads to the de-coupled quadratic equations; moreover, when damping is considered, it leads to a set of coupled quadratic equations. According to the quadratic form of the algebraic equations at each time step, a novel mathematical technique called Elimination of Discontinuous Velocities is presented to detect the real velocity in every instance.

In this study, shear frames are selected to illustrate the proposed method. The method largely eliminates the disadvantages of other methods such as mathematical complexity and time-consuming calculation of a modal matrix in large-order structures, as well as stability concerns and adjustment of the analytical coefficients in numerical integration methods. It should be noted that it is possible to extend this approach to other multi-dimensional structures, too. Furthermore, while we assume that the structure will behave linearly in this investigation, it is possible for the proposed method to be used for nonlinear analyses in future studies by making certain simple modifications.

\section{Force versus energy equations}

In this section, shear frames are introduced in brief. Subsequently, the mechanical energy relationships of these systems are expressed and, by applying the principle of conservation of energy, the equations of motion are derived from energy balance relationships of the system. The principal objective of these relations is to prove the equivalence of the force and energy approaches in structural dynamics. Finally, at the end of this section, the advantages and disadvantages of applying these methods are compared with each other.

Figure 1 depicts an $n$-story shear frame (or shear building) as one of the simplest MDF systems that is widely used in civil engineering. In this idealization, the beams and floor systems are rigid in flexure, and several factors such as axial deformation of the beams and columns and the effect of axial force on the stiffness of the columns are neglected [8]. In this respect, the deflected building shares many features of a cantilever 


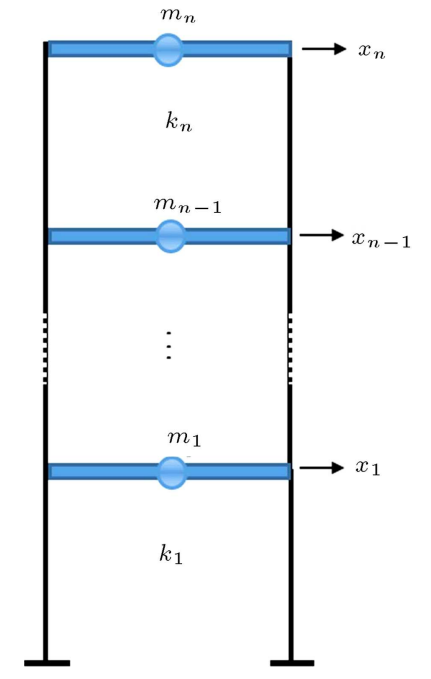

Figure 1. Shear-frame structure.

beam that is deflected by shear force only, hence the name shear building [43], where $x_{i}$ denotes the displacement of the $i$ th story. Moreover, $k_{i}$ and $m_{i}$ are the stiffness and mass of the $i$ th story, respectively. For these structures, the potential energy of the system $\left(E_{P}\right)$ can be expressed below by assuming a linear relationship between force and displacement.

$$
\begin{aligned}
E_{K}= & \frac{1}{2} m_{1} v_{1}^{2}+\frac{1}{2} m_{2} v_{2}^{2}+\cdots+\frac{1}{2} m_{i} v_{i}^{2}+\cdots \\
& +\frac{1}{2} m_{n-1} v_{n-1}^{2}+\frac{1}{2} m_{n} v_{n}^{2}
\end{aligned}
$$

Moreover, the kinetic energy of the structure regarding $v_{i}=d x_{i} / d t$ (the velocity of the $i$ th mass) is given by the following:

$$
E_{T}=\frac{1}{2} k_{1}\left(x_{1}\right)^{2}+\sum_{i=2}^{n} \frac{1}{2} k_{i}\left(x_{i}-x_{i-1}\right)^{2}+\sum_{i=1}^{n} \frac{1}{2} m_{i} v_{i}^{2}
$$

By neglecting the effects of energy dissipations and using the summation notation, the total energy of the system $\left(E_{T}\right)$ (the sum of the potential and kinetic energies) can be written as follows:

$$
E_{T}=\frac{1}{2} k_{1}\left(x_{1}\right)^{2}+\sum_{i=2}^{n} \frac{1}{2} k_{i}\left(x_{i}-x_{i-1}\right)^{2}+\sum_{i=1}^{n} \frac{1}{2} m_{i} v_{i}^{2} .
$$

From a physical perspective, the law of conservation of energy states that the total energy of an isolated system remains constant, which is said to be conserved over time [44]. Hence, differentiating Eq. (3) with respect to time, we obtain:

$$
\frac{d E_{T}}{d t}=0
$$

alternatively:

$$
k_{1} x_{1} v_{1}+\sum_{i=2}^{n} k_{i}\left(x_{i}-x_{i-1}\right)\left(v_{i}-v_{i-1}\right)+\sum_{i=1}^{n} m_{i} v_{i} a_{i}=0
$$

where $a_{i}$ is the acceleration of the $i$ th mass, i.e.:

$$
a_{i}=\frac{d v_{i}}{d t}
$$

Expanding the series in Eq. (5) leads to:

$$
\begin{aligned}
k_{1} x_{1} v_{1} & +k_{2}\left(x_{2}-x_{1}\right) v_{2}+k_{2}\left(x_{1}-x_{2}\right) v_{1} \\
& +k_{3}\left(x_{3}-x_{2}\right) v_{3}+k_{3}\left(x_{2}-x_{3}\right) v_{2}+\cdots \\
& +m_{1} v_{1} a_{1}+m_{2} v_{2} a_{2}+m_{3} v_{3} a_{3}+\cdots=0 .
\end{aligned}
$$

By factoring $v_{1}, v_{2}, v_{3}, \cdots$, one can write:

$$
\begin{aligned}
v_{1}\left[k_{1} x_{1}\right. & \left.+k_{2}\left(x_{1}-x_{2}\right)+m_{1} a_{1}\right] \\
& +v_{2}\left[k_{2}\left(x_{2}-x_{1}\right)+m_{2} a_{2}\right]+\cdots \\
& +v_{n}\left[k_{n}\left(x_{n}-x_{n-1}\right)+m_{n} a_{n}\right]=0,
\end{aligned}
$$

which corresponds to the following matrix form:

$$
\begin{aligned}
& {\left[\begin{array}{cccc}
m_{1} & 0 & 0 & 0 \\
0 & m_{2} & 0 & 0 \\
0 & 0 & \ddots & 0 \\
0 & 0 & 0 & m_{n}
\end{array}\right]\left\{\begin{array}{c}
\ddot{x}_{1} \\
\ddot{x}_{2} \\
\vdots \\
\ddot{x}_{n}
\end{array}\right\}} \\
& +\left[\begin{array}{cccc}
k_{1}+k_{2} & -k_{2} & 0 & 0 \\
-k_{2} & k_{2}+k_{3} & -k_{3} & 0 \\
0 & -k_{3} & \ddots & -k_{n} \\
0 & 0 & -k_{n} & k_{n}
\end{array}\right]\left\{\begin{array}{c}
x_{1} \\
x_{2} \\
\vdots \\
x_{n}
\end{array}\right\}=\left\{\begin{array}{c}
0 \\
0 \\
\vdots \\
0
\end{array}\right\}
\end{aligned}
$$

As is clear, Eq. (9) represents the dynamic force equilibrium equations of an $n$-story shear frame and, as previously mentioned, the primary objective of this part is the proof of the equality of energy and force balance equations. As a result, it can be noted that force equilibrium equations can be obtained from the derivative of energy equations and, mutually, energy equations might be derived from the integration of force balance equations. It must be stated that although these two equations are basically the same, each of them has its own advantages and disadvantages in practice. For illustration, see Table 1.

Here, it is important to note that the presented method in this study includes the linear behavior of shear frames (as a simple structural system). In other words, the nonlinear response analysis of general structures like systems with hysteresis does not fall within the scope of this work. However, the idea presented in this research can be the basis for the ultimate goal of dynamic analysis of large-scale structures with different types of nonlinearities. 
Table 1. Comparison of the force and energy equilibrium equations mathematically.

\begin{tabular}{lll}
\hline Type of equations & \multicolumn{1}{c}{ Advantages } & \multicolumn{1}{c}{ Disadvantages } \\
\hline \multirow{2}{*}{ Force equilibrium } & $\begin{array}{l}\text { Quadratic (non-linear) terms do not exist in } \\
\text { these equations* }\end{array}$ & $\begin{array}{l}\text { Second order of derivative in the equations } \\
\text { that leads to an increase in the number of } \\
\text { unknowns including displacement, velocity, } \\
\end{array}$ \\
Energy equilibrium & $\begin{array}{l}\text { First order of derivative in the equations that } \\
\text { leads to reducing the number of unknowns, } \\
\text { including: displacement, velocity }\end{array}$ & Existence of quadratic (non-linear) terms \\
\hline
\end{tabular}

*: Except in nonlinear analysis

\section{Methodology}

\subsection{Derivation of discretized energy balance scheme}

Now, the energy balance approach is extended for general forced vibration problems to include the effects of damping, in which damping is assumed to be linear regarding velocity (viscous damping) in this study. For this purpose, consider the equations of motion of an $n$-DOF shear frame as follows:

$$
\begin{aligned}
& m_{1} \ddot{x}_{1}+\left(c_{1}+c_{2}\right) \dot{x}_{1}-c_{2} \dot{x}_{2}+\left(k_{1}+k_{2}\right) x_{1}-k_{2} x_{2}=p_{1}, \\
& m_{2} \ddot{x}_{2}+\left(c_{2}+c_{3}\right) \dot{x}_{2}-c_{2} \dot{x}_{1}-c_{3} \dot{x}_{3}+\left(k_{2}+k_{3}\right) x_{2} \\
& -k_{2} x_{1}-k_{3} x_{3}=p_{2}, \\
& m_{i} \ddot{x}_{i}+\left(c_{i}+c_{i+1}\right) \dot{x}_{i}-c_{i} \dot{x}_{i-1}-c_{i+1} \dot{x}_{i+1} \\
& +\left(k_{i}+k_{i+1}\right) x_{i}-k_{i} x_{i-1}-k_{i+1} x_{i+1}=p_{i}, \\
& \vdots \\
& m_{n-1} \ddot{x}_{n-1}+\left(c_{n-1}+c_{n}\right) \dot{x}_{n-1}-c_{n-1} \dot{x}_{n-2}-c_{n} \dot{x}_{n} \\
& +\left(k_{n-1}+k_{n}\right) x_{n-1}-k_{n-1} x_{n-2}-k_{n} x_{n}=p_{n-1} \\
& m_{n} \ddot{x}_{n}+c_{n} \dot{x}_{n}-c_{n} \dot{x}_{n-1}+k_{n} x_{n}-k_{n} x_{n-1}=p_{n},
\end{aligned}
$$

where $c_{i}$ and $p_{i}$ denote the damping coefficient and external force of the $i$ th mass, respectively.

Integrating the $i$ th equation of Eq. (10) with respect to $x_{i}$, we get:

$$
\begin{aligned}
\int\left(m_{i} \ddot{x}_{i}\right. & +\left(c_{i}+c_{i+1}\right) \dot{x}_{i}-c_{i} \dot{x}_{i-1}-c_{i+1} \dot{x}_{i+1} \\
& \left.+\left(k_{i}+k_{i+1}\right) x_{i}-k_{i} x_{i-1}-k_{i+1} x_{i+1}\right) d x_{i} \\
& =\int p_{i} d x_{i} .
\end{aligned}
$$

Each part of Eq. (11) according to the definition of various energies, i.e., the area under the curve of the load-displacement, implies the changes in a specific type of energy.

$$
\begin{aligned}
\underbrace{\int\left[m_{i} \ddot{x}_{i}\right] d x_{i}}_{\Delta E_{K}} & +\underbrace{\int\left[\left(c_{i}+c_{i+1}\right) \dot{x}_{i}-c_{i} \dot{x}_{i-1}-c_{i+1} \dot{x}_{i+1}\right] d x_{i}}_{\Delta E_{D}} \\
& +\underbrace{\int\left[\left(k_{i}+k_{i+1}\right) x_{i}-k_{i} x_{i-1}-k_{i+1} x_{i+1}\right] d x_{i}}_{\Delta E_{P}} \\
= & \underbrace{\int p_{i} d x_{i}}_{\Delta E_{F}} .
\end{aligned}
$$

The first integral in the Left Hand Side (LHS) represents the changes of kinetic energy $\Delta E_{k}$ and, by the definition of velocity, it takes the following form:

$$
\Delta E_{K}=\int\left[m_{i} \ddot{x}_{i}\right] d x_{i} \stackrel{\ddot{x}_{i} d x_{i}=v_{i} d v_{i}}{\longrightarrow} \int m_{i} v_{i} d v_{i} .
$$

Integration from zero to arbitrary time gives:

$$
\Delta E_{K}=\frac{1}{2} m_{i} v_{i}^{2}-\frac{1}{2} m_{i} v_{i(0)}^{2} .
$$

The second integral on the left-hand side expresses the changes in damped energy $\left(\Delta E_{D}\right)$, which is sometimes also called the energy loss, that is:

$$
\Delta E_{D}=\int\left[\left(c_{i}+c_{i+1}\right) \dot{x}_{i}-c_{i} \dot{x}_{i-1}-c_{i+1} \dot{x}_{i+1}\right] d x_{i} .
$$

Based on the definition of velocity, Eq. (15) can also be written as follows:

$$
\Delta E_{D}=\int_{0}^{t}\left[\left(c_{i}+c_{i+1}\right) v_{i}^{2}-c_{i} v_{i-1} v_{i}-c_{i+1} v_{i+1} v_{i}\right] d t
$$


In addition, the change in potential energy $\left(\Delta E_{P}\right)$ is:

$$
\Delta E_{P}=\int\left[\left(k_{i}+k_{i+1}\right) x_{i}-k_{i} x_{i-1}-k_{i+1} x_{i+1}\right] d x_{i}
$$

By operations equivalent to Eq. (17), it can be shown that:

$$
\Delta E_{P}=\int_{0}^{t}\left[\left(k_{i}+k_{i+1}\right) x_{i}-k_{i} x_{i-1}-k_{i+1} x_{i+1}\right] v_{i} d t
$$

and, eventually, the changes in energy of the external loads $\Delta E_{F}$ are given by:

$$
\Delta E_{F}=\int p_{i} d x_{i}
$$

Similarly, in terms of velocity, the aforementioned energy in Eq. (19) becomes:

$$
\Delta E_{F}=\int_{0}^{t} p_{i} v_{i} d t
$$

Therefore, the energy balance equation for the $i$ th mass is given as follows:

$$
\Delta E_{k}+\Delta E_{D}+\Delta E_{P}=\Delta E_{F} .
$$

Here, energy balance equations are written for all of the masses:

$$
\begin{aligned}
\frac{1}{2} m_{1} v_{1}^{2} & -\frac{1}{2} m_{1} v_{1(0)}^{2}+\int_{0}^{t}\left[\left(c_{1}+c_{2}\right) v_{1}^{2}-c_{2} v_{2} v_{1}\right] d t \\
& +\int_{0}^{t}\left[\left(k_{1}+k_{2}\right) x_{1}-k_{2} x_{2}\right] v_{1} d t=\int_{0}^{t}\left[p_{1} v_{1}\right] d t \\
\frac{1}{2} m_{2} v_{2}^{2}- & \frac{1}{2} m_{2} v_{2(0)}^{2} \\
& +\int_{0}^{t}\left[\left(c_{2}+c_{3}\right) v_{2}^{2}-c_{2} v_{1} v_{2}-c_{3} v_{3} v_{2}\right] d t \\
& +\int_{0}^{t}\left[\left(k_{2}+k_{3}\right) x_{2}-k_{2} x_{1}-k_{3} x_{3}\right] v_{2} d t \\
\frac{1}{2} m_{i} v_{i}^{2}- & \frac{1}{2} m_{i} v_{i(0)}^{2} \\
= & \int_{0}^{t}\left[p_{2} v_{2}\right] d t
\end{aligned}
$$

$$
\begin{aligned}
& +\int_{0}^{t}\left[\left(c_{i}+c_{i+1}\right) v_{i}^{2}-c_{i} v_{i-1} v_{i}-c_{i+1} v_{i+1} v_{i}\right] d t \\
& +\int_{0}^{t}\left[\left(k_{i}+k_{i+1}\right) x_{i}-k_{i} x_{i-1}-k_{i+1} x_{i+1}\right] v_{i} d t \\
& =\int_{0}^{t}\left[p_{i} v_{i}\right] d t
\end{aligned}
$$$$
\vdots
$$

$$
\begin{aligned}
& \frac{1}{2} m_{n-1} v_{n-1}^{2}-\frac{1}{2} m_{n-1} v_{n-1(0)}^{2}+\int_{0}^{t}\left[\left(c_{n-1}+c_{n}\right) v_{n-1}^{2}\right. \\
& \left.\quad-c_{n-1} v_{n-2} v_{n-1}-c_{n} v_{n} v_{n-1}\right] d t \\
& \quad+\int_{0}^{t}\left[\left(k_{n-1}+k_{n}\right) x_{n-1}-k_{n-1} x_{n-2}-k_{n} x_{n}\right] v_{n-1} d t \\
& =\int_{0}^{t}\left[p_{n-1} v_{n-1}\right] d t,
\end{aligned}
$$$$
\frac{1}{2} m_{n} v_{n}^{2}-\frac{1}{2} m_{n} v_{n(0)}^{2}+\int_{0}^{t}\left[c_{n} v_{n}^{2}-c_{n} v_{n-1} v_{n}\right] d t
$$$$
+\int_{0}^{t}\left[k_{n} x_{n}-k_{n} x_{n-1}\right] v_{n} d t=\int_{0}^{t}\left[p_{n} v_{n}\right] d t
$$

Considering the $i$ th mass:

$$
\begin{aligned}
\frac{1}{2} m_{i} v_{i}^{2} & -\frac{1}{2} m_{i} v_{i(0)}^{2} \\
& +\int_{0}^{t}\left[\left(c_{i}+c_{i+1}\right) v_{i}^{2}-c_{i} v_{i-1} v_{i}-c_{i+1} v_{i+1} v_{i}\right] d t \\
& +\int_{0}^{t}\left[\left(k_{i}+k_{i+1}\right) x_{i}-k_{i} x_{i-1}-k_{i+1} x_{i+1}\right] v_{i} d t \\
= & \int_{0}^{t} p_{i} v_{i} d t .
\end{aligned}
$$

In principle, after discretizing Eq. (23) by using numerical integration methods such as Trapezoidal and Simpson techniques [12], the correspondent energy 
equation of the $i$ th mass would be evaluated through Eq. (24) (See Appendix A, for details).

$$
\begin{aligned}
A_{i} v_{i(j \Delta t)}^{2} & +B_{i} v_{i-1(j \Delta t)} v_{i(j \Delta t)}+C_{i} v_{i+1(j \Delta t)} v_{i(j \Delta t)} \\
& +D_{i} v_{i(j \Delta t)}+E_{i}=0
\end{aligned}
$$

where $A_{i}, B_{i}, C_{i}, D_{i}$, and $E_{i}$ are the constant coefficients that are determined by discretizing integrals in energy balance relations; in the first time step where the Trapezoidal method is used, these coefficients take the following forms:

$$
\begin{aligned}
A_{i}= & 0.5 m_{i}+0.5 \Delta t\left(c_{i}+c_{i+1}\right) \\
B_{i}= & -0.5 \Delta t \cdot c_{i}, \quad C_{i}=-0.5 \Delta t \cdot c_{i+1}, \\
D_{i}= & 0.5 \Delta t\left[\left(k_{i}+k_{i+1}\right) x_{i(\Delta t)}-k_{i} \cdot x_{i-1(\Delta t)}\right. \\
& -k_{i+1} \cdot x_{i+1(\Delta t)}-p_{i(\Delta t)]}, \\
E_{i}= & -0.5 m_{i} v_{i(0)}^{2}+0.5 \Delta t \cdot v_{i(0)}\left[\left(c_{i}+c_{i+1}\right) v_{i(0)}\right. \\
& -c_{i} \cdot v_{i-1(0)}+c_{i+1} \cdot v_{i+1(0)}+\left(k_{i}+k_{i+1}\right) x_{i(0)} \\
& \left.-k_{i} x_{i-1(0)}-k_{i+1} x_{i+1(0)}-p_{i(0)}\right] .
\end{aligned}
$$

In the time steps after the primary time step, to increase the accuracy of integration by using the Simpson method, one can write:

$$
\begin{aligned}
A_{i}= & 0.5 m_{i}+(\Delta t / 3)\left(c_{i}+c_{i+1}\right) \\
B_{i}= & -(\Delta t / 3) \cdot c_{i}, \quad C_{i}=-(\Delta t / 3) \cdot c_{i+1}, \\
D_{i}= & (\Delta t / 3)\left[\left(k_{i}+k_{i+1}\right) x_{i(j \Delta t)}-k_{i} \cdot x_{i-1(j \Delta t)}\right. \\
& -k_{i+1} \cdot x_{i+1(j \Delta t)}-p_{i(j \Delta t)]} \\
E_{i}= & -0.5 m_{i} v_{i(0)}^{2}+(\Delta t / 3)\left\{v _ { i ( 0 ) } \left[\left(c_{i}+c_{i+1}\right) v_{i(0)}\right.\right. \\
& -c_{i} \cdot v_{i-1(0)}+c_{i+1} \cdot v_{i+1(0)}+\left(k_{i}+k_{i+1}\right) x_{i(0)} \\
& \left.-k_{i} x_{i-1(0)}-k_{i+1} x_{i+1(0)}-p_{i(0)}\right] \\
& +4 v_{i(\Delta t)}\left[\left(c_{i}+c_{i+1}\right) v_{i(\Delta t)}-c_{i} \cdot v_{i-1(\Delta t)}\right. \\
& +c_{i+1} \cdot v_{i+1(\Delta t)}+\left(k_{i}+k_{i+1}\right) x_{i(\Delta t)} \\
& \left.-k_{i} x_{i-1(\Delta t)}-k_{i+1} x_{i+1(\Delta t)}-p_{i(\Delta t)}\right] \\
& +2 v_{i(2 \Delta t)}\left[\left(c_{i}+c_{i+1}\right) v_{i(2 \Delta t)}-c_{i} \cdot v_{i-1(2 \Delta t)}\right. \\
& +c_{i+1} . v_{i+1(2 \Delta t)}+\left(k_{i}+k_{i+1}\right) x_{i(2 \Delta t)}
\end{aligned}
$$

$$
\begin{aligned}
& \left.-k_{i} x_{i-1(2 \Delta t)}-k_{i+1} x_{i+1(2 \Delta t)}-p_{i(2 \Delta t)}\right] \\
& +\cdots+v_{i(j \Delta t)}\left[\left(c_{i}+c_{i+1}\right) v_{i(j \Delta t)}-c_{i} \cdot v_{i-1(j \Delta t)}\right. \\
& +c_{i+1} \cdot v_{i+1(j \Delta t)}+\left(k_{i}+k_{i+1}\right) x_{i(j \Delta t)} \\
& \left.\left.-k_{i} x_{i-1(j \Delta t)}-k_{i+1} x_{i+1(j \Delta t)}-p_{i(j \Delta t)}\right]\right\}, \quad \text { (26) }
\end{aligned}
$$

where $j$ denotes the number of steps.

\subsection{Solution procedure of coupled quadratic energy equations}

As observed earlier in the previous section, after discretizing the energy balance equations, a set of equations in the following quadratic form is obtained:

$$
\begin{aligned}
& a_{1} v_{1}^{2}+c_{1} v_{2} v_{1}+d_{1} v_{1}+e_{1}=0 \\
& a_{2} v_{2}^{2}+b_{2} v_{1} v_{2}+c_{2} v_{3} v_{1}+d_{2} v_{2}+e_{2}=0 \\
& \vdots \\
& a_{i} v_{i}^{2}+b_{i} v_{i-1} v_{i}+c_{i} v_{i+1} v_{i}+d_{i} v_{i}+e_{i}=0 \\
& \vdots \\
& a_{n-1} v_{n-1}^{2}+b_{n-1} v_{n-2} v_{n-1}+c_{n-1} v_{n} v_{n-1} \\
& \quad+d_{n-1} v_{n-1}+e_{n-1}=0, \\
& a_{n} v_{n}^{2}+b_{n} v_{n-1} v_{n}+d_{n} v_{n}+e_{n}=0 .
\end{aligned}
$$

Mathematically, in solving the previous equations, there are two main problems:

A) These equations are coupled, meaning that they are dependent on each other and must be solved simultaneously; in other words, one cannot calculate $v_{i}$ through the $i$ th equation directly. In addition, it should be noted that, in the absence of damping, the equations would be de-coupled, viz., damping is the reason for coupling the equations;

B) The quadratic form of equations implies more than one velocity at each time step; in other words, from a physical perspective at every time step, these relations provide some unreal velocities in addition to the actual velocity of the structure.

To better understand the above expressions, suppose that, in a sample 2-DOF structure in a given time step, a mathematical equation of the form is to be solved as follows:

$$
\left\{\begin{array}{l}
v_{1}^{2}+v_{2} v_{1}+v_{1}+1=0 \\
2 v_{2}^{2}+v_{2} v_{1}+v_{2}+2=0
\end{array}\right.
$$

If the terms of $v_{2} v_{1}$ do not exist, one can obtain two values of each of $v_{1}$ and $v_{2}$ by solving the two 
uncoupled quadratic equations. However, given $v_{2} v_{1}$, by combining the equations together and writing them only in terms of one variable, we have:

$$
\left\{\begin{array}{l}
v_{1}^{4}+2 v_{1}^{3}+6 v_{1}^{2}+3 v_{1}+2=0 \\
2 v_{2}^{4}+v_{2}^{3}+8 v_{2}^{2}+2 v_{1}+4=0
\end{array}\right.
$$

From Eq. (29), by solving the two fourth-degree equation, it is apparent that four values for each of $v_{1}$ and $v_{2}$ will be obtained. Note that, at any moment, the velocity of each mass is unique and there is only one value for the real velocity of the system, while, in this case, three unrealistic velocities for each mass have appeared in the equation. Apparently, this method (direct method) cannot be used to calculate the velocities at any instants, especially in large DOF systems; therefore, this study aims to apply a numerical method to calculate the velocities in each time step. In this context, as demonstrated in Appendix B, well-known solution techniques, such as the NewtonRaphson method, are not efficient for the system of equations under consideration in this study. Two main reasons for the inefficiency of these methods when applied to the considered equations in each time step can be expressed as follows:

a) The need for the derivative of the system of equations;

b) Complex and time-consuming process of inverting the Jacobi matrix (specifically in large-scale structures).

\subsection{Elimination of discontinuous velocity technique}

The problem of coupled equations exists in many engineering fields, particularly in multi-dimensional systems; hence, many researchers have studied how to solve these equations (e.g., refer to [45-51]). For the current study, a novel numerical method is presented in which the real velocities of the system at any time step can be easily calculated by removing the unrealistic velocities from the coupled equations.

In the proposed technique, first, the problem of coupled equations is resolved by neglecting the coupling terms (terms that are the product of two different velocities). In this case, by assuming a structure with $n$-DOF, an $n$-quadratic equation is given in terms of velocity in this study. To solve the problem of non-linear equations (quadratic in terms of velocity) and detect the actual velocities of the system at any time, it is assumed that the variation of velocities with respect to time is continuous. Therefore, among the two velocities obtained at any time from the quadratic equations, the velocity closer to that of the previous time step is selected as the real velocity of the structure. Therefore, the name of the method is chosen as Elimination of Discontinuous Velocities Technique. At the beginning of this procedure, the coupling terms are ignored to obtain the velocities. Herein, the values of continuous velocities are substituted into them, and this iteration will carry on until the velocities in two subsequent iterations approach each other. Table 2 gives a summary of the method.

\section{Numerical examples and results}

Various examples of multi-story shear-frame structures are analyzed by using the energy method in this section. In the first example, the vibration of a simple twostory shear building has been investigated to describe the procedure of the presented method in detail. In the next examples, some multi-story shear-frame structures subjected to harmonic and earthquakes loadings have been studied. Moreover, the results are compared with the exact solution and other common methods.

Example 4.1. The free damped vibration of a two-story shear building. Figure 2 shows a 2DOF shear frame where, for convenience, the dynamic properties of the structure are chosen as follows: $m_{1}=$ $m_{2}=k_{1}=k_{2}=1 ; c_{1}=0.06, c_{2}=0.16$ (all units are assumed to be compatible). Moreover, the following initial conditions will be considered in this example:

$$
x_{0}=\left\{\begin{array}{l}
1 \\
2
\end{array}\right\}, \quad v_{0}=\left\{\begin{array}{l}
3 \\
4
\end{array}\right\} .
$$

In free-vibration cases, the equation of motion of these structures can be expressed as follows:

$$
[m]\{\ddot{x}\}+[c]\{\dot{x}\}+[k]\{x\}=\{0\},
$$

where the mass, damping, and stiffness matrices are given below:

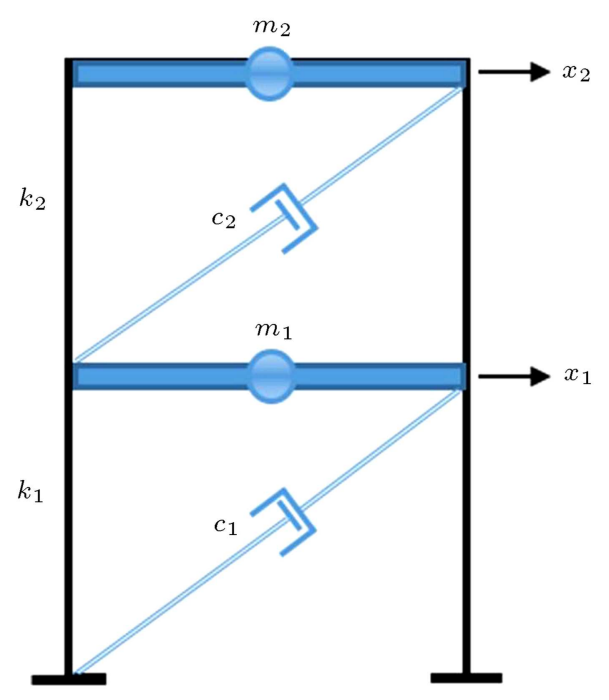

Figure 2. Free vibration of a two-story shear frame. 
Table 2. Summary of the step-by-step solution procedure of the presented method.
A. Initial calculations:
1. Form dynamic matrices: mass $m$, damping $c$, and stiffness $k$
2. Form the vectors of initial conditions: initial displacements $x_{0}$ and velocities $v_{0}$
3. Select the time step $\Delta t$
4. Select the tolerance for each iteration $e=10^{-s}$ ( $s$ is a positive integer number)

\section{B. For each time step:}

5. Calculate a starting vector for $x_{i}$ and displacement vector at the time of $t=i \Delta t$

$$
x_{i}^{(1)}=x_{0}+d x^{(1)}, \quad d x^{(1)}=v_{0} \Delta t
$$

(the superscripts and subscripts refer to the number of iteration and time step, respectively)

6. Calculate the coefficient of energy balance equation, i.e., $A_{i}, B_{i}, C_{i}, D_{i}$, for all masses. Note that the trapezoidal rule for the first time step and, subsequently, Simpson rule must be used.

7. Neglect the coupling terms, i.e., $B_{i}=C_{i}=0$

8. Solve the quadratic equation of energy balance for the velocity of the $i$ th mass using the corresponding $\Delta_{i}$ and $v_{i}=(-D \pm \Delta) / 2 A$

9. Select a velocity that is closer to the previous time step (call it $v_{i}$ )

(Elimination of Discontinuous Velocities)

10. Calculate a new approximated vector for $\mathbf{x}_{\mathbf{i}}$ by using the average of new obtained velocities and initial velocities

$$
x_{i}^{(j)}=x_{i-1}+d x^{(j)}, \quad d x^{(j)}=0.5\left(v_{i-1}+v_{i}\right) \Delta t, \quad j=2,3, \cdots
$$

11. Determine the coupling terms, neglected at first

12. Iterate through steps 6 to 11 , except step 7 , to reach convergence

13. Continue the procedure for subsequent time steps

$$
\begin{aligned}
& {[m]=\left[\begin{array}{ll}
1 & 0 \\
0 & 1
\end{array}\right], \quad[c]=\left[\begin{array}{cc}
0.22 & -0.16 \\
-0.16 & 0.16
\end{array}\right],} \\
& {[k]=\left[\begin{array}{cc}
2 & -1 \\
-1 & 1
\end{array}\right] .}
\end{aligned}
$$

Thus, by multiplying the matrices and vectors, there are the following governing equations:

$$
\left\{\begin{array}{l}
\ddot{x}_{1}+0.22 \dot{x}_{1}-0.16 \dot{x}_{2}+2 x_{1}-x_{2}=0 \\
\ddot{x}_{2}-0.16 \dot{x}_{1}+0.16 \dot{x}_{2}-x_{1}+x_{2}=0
\end{array}\right.
$$

Herein, the Laplace transform method is used to determine the exact solution of this problem (for details, see Appendix C).

$$
\left\{\begin{aligned}
x_{1}= & -0.336 e^{-0.1733 t} \cos (1.608 t+0.681) \\
& +4.445 e^{-0.0167 t} \cos (0.618 t-1.283) \\
x_{2}= & 0.210 e^{-0.1733 t} \cos (1.608 t+0.753) \\
& +7.180 e^{-0.0167 t} \cos (0.618 t-1.3106)
\end{aligned}\right.
$$

\subsection{Applying the energy method}

By substituting the assumed parameters of this example into Eq. (23), the energy balance equations of the system are given below:

$$
\left\{\begin{array}{c}
0.5 v_{1}^{2}-0.5 v_{1(0)}^{2}+\int_{0}^{t}\left[0.22 v_{1}^{2}-0.16 v_{2} v_{1}\right] d t \\
+\int_{0}^{t}\left[2 x_{1}-x_{2}\right] v_{1} d t=0 \\
0.5 v_{2}^{2}-0.5 v_{2(0)}^{2}+\int_{0}^{t}\left[0.16 v_{2}^{2}-0.16 v_{1} v_{2}\right] d t \\
+\int_{0}^{t}\left[x_{2}-x_{1}\right] v_{2} d t=0
\end{array}\right.
$$

Now, the integrals in the above equations should be discretized to obtain the algebraic equations. Since Simpson rule needs at least three points for integration, it cannot be used in the first time step. Hence, the trapezoidal method must be applied in the first time step. For the problem at hand, the size of time intervals is assumed to be $\Delta t=0.1 \mathrm{~s}$ and, according to Table 2, $x_{1}$ and $x_{2}$ (dynamic responses of floors at the time of $t=0.1 \mathrm{~s}$ ) would be approximated by the Euler formula 
as follows:

$$
\left\{\begin{array}{l}
x_{1(0.1)} \approx x_{1(0)}+v_{1(0)} \times \Delta t=1.3 \\
x_{2(0.1)} \approx x_{2(0)}+v_{2(0)} \times \Delta t=2.4
\end{array}\right.
$$

The discretized form of Eq. (36) is given below:

$$
\left\{\begin{array}{l}
0.511 v_{1}^{2}-0.008 v_{2} v_{1}+0.01 v_{1}-4.497=0 \\
0.508 v_{2}^{2}-0.008 v_{2} v_{1}+0.055 v_{2}-7.768=0
\end{array}\right.
$$

By neglecting the coupling term, i.e., $\left(-0.008 v_{1} v_{2}\right)$, two quadratic equations are given as follows:

$$
\left\{\begin{array}{l}
0.511 v_{1}^{2}+0.01 v_{1}-4.497=0 \\
0.508 v_{2}^{2}+0.055 v_{2}-7.768=0
\end{array}\right.
$$

Roots of these quadratic equations are:

$$
\left\{\begin{array}{l}
0.511 v_{1}^{2}+0.01 v_{1}-4.497=0 \rightarrow v_{1}=2.9568 \\
\quad v_{1}=-2.9763 \\
0.508 v_{2}^{2}+0.055 v_{2}-7.768=0 \rightarrow v_{2}=3.5867 \\
v_{2}=-3.9649
\end{array}\right.
$$

By comparing the roots obtained from Eq. (39) with the velocity in the previous step, $v_{1}=3, v_{2}=4$, the closest velocities to the previous step are selected and others are omitted:

$$
\left\{\begin{array}{l}
0.511 v_{1}^{2}+0.01 v_{1}-4.497=0 \\
\rightarrow v_{1}=2.9568, \quad v_{1}=-2.9763 \times \\
0.508 v_{2}^{2}+0.055 v_{2}-7.768=0 \\
\rightarrow v_{2}=3.5867, \quad v_{2}=-3.9649 \times
\end{array}\right.
$$

Now, new values $x_{1}$ and $x_{2}$ at $t=0.1 \mathrm{~s}$ can be approximated by using the average of the velocity of this step and the previous step; hence:

$$
\left\{\begin{array}{l}
x_{1} \approx x_{1(0)}+\frac{v_{1(0)}+v_{1(0.1)}}{2} \times \Delta t=1.2978 \\
x_{2} \approx x_{2(0)}+\frac{v_{2(0)}+v_{2(0.1)}}{2} \times \Delta t=2.3928
\end{array}\right.
$$

Here, the coupling term $\left(-0.008 v_{1} v_{2}\right)$, which was neglected previously, might be given by the substitution of $v_{1}=2.9568$ and $v_{2}=3.5867$.

$$
v_{1}=2.9568, v_{2}=3.5867 \rightarrow-0.008 v_{1} v_{2}=-0.0848 \text {. }
$$

Moreover, new values of velocities of the system can be determined as follows:

$$
\begin{gathered}
\left\{\begin{array}{l}
0.511 v_{1}^{2}-0.008 v_{2} v_{1}+0.01 v_{1}-4.497=0 \\
0.508 v_{2}^{2}-0.008 v_{2} v_{1}+0.055 v_{1}-7.768=0
\end{array}\right. \\
\stackrel{-0.008 v_{1} v_{2}=-0.0848}{\longrightarrow}\left\{\begin{array}{l}
0.511 v_{1}^{2}+0.01 v_{1}-4.5882=0 \\
0.508 v_{2}^{2}+0.055 v_{1}-7.8592=0
\end{array}\right.
\end{gathered}
$$

Similarly, real velocities are given below:

$$
\left\{\begin{array}{l}
0.511 v_{1}^{2}+0.01 v_{1}-4.5882=0 \rightarrow v_{1}=2.9867 \\
v_{1}=-3.0063 \times \\
0.508 v_{2}^{2}+0.055 v_{1}-7.8592=0 \rightarrow v_{2}=3.8795 \\
v_{2}=-3.9878 \times
\end{array}\right.
$$

Now, the updated coupling term becomes:

$$
v_{1}=2.9867, v_{2}=3.8795 \rightarrow-0.008 v_{1} v_{2}=-0.0927 \text {. }
$$

A relative error, $e_{i}^{j}$, for velocities as an absolute value of $\left(v_{i}^{j}-v_{i}^{j-1}\right) / v_{i}^{j-1}$ is introduced for the convergence criterion, where $i$ and $j$ represent the number of stories and iterations, respectively. As shown in Table 3, the procedure can be monitored better by this definition. Note that tolerance is chosen as $10^{-2}$ in this case. Moreover, Figure 3 illustrates the process of convergence, showing the error of analysis versus number of iterations.

If a computer program is used to continue the process to $t=10 \mathrm{~s}$, the dynamic response of the system can be obtained, as shown in Figure 4. This figure compares the obtained results of the presented method against the exact solution of the problem. As shown in Figure 4 , although the size of time intervals $\Delta t=0.1$ selected is not very small in this analysis, it can be seen that the proposed method has excellent accuracy compared with the exact solution; in other words, the numerical solution can properly approach the exact solution of the problem in this case.

Now, by choosing a fixed time interval, which is deliberately not chosen too small, typically $\Delta t=0.2$

Table 3. Convergence of velocities in the first step.

\begin{tabular}{ccccccc}
\hline $\begin{array}{c}\text { Number } \\
\text { of } \\
\text { iterations }\end{array}$ & $\boldsymbol{v}_{\mathbf{1}}$ & $\boldsymbol{e}_{\mathbf{1}}$ & $\boldsymbol{v}_{\mathbf{2}}$ & $\boldsymbol{e}_{\mathbf{2}}$ & $\boldsymbol{e}_{\max }<\mathbf{0 . 0 1}$ \\
\hline 1 & 2.9568 & 0.0144 & 3.5867 & $\mathbf{0 . 0 3 5 8}$ & $\times$ \\
2 & 2.9867 & 0.0044 & 3.8795 & $\mathbf{0 . 0 3 0 1}$ & $\times$ \\
3 & 2.9872 & $\mathbf{0 . 0 0 0 1 6}$ & 3.8799 & 0.00009 & $\sqrt{ }$ \\
\hline
\end{tabular}

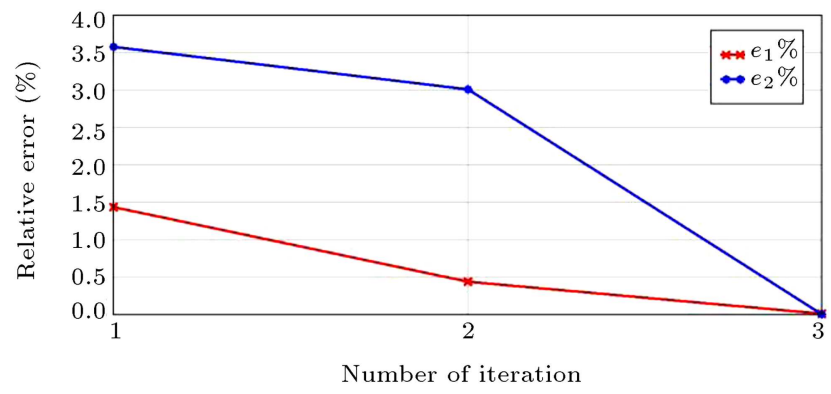

Figure 3. The process of convergence in Example 4.1. 


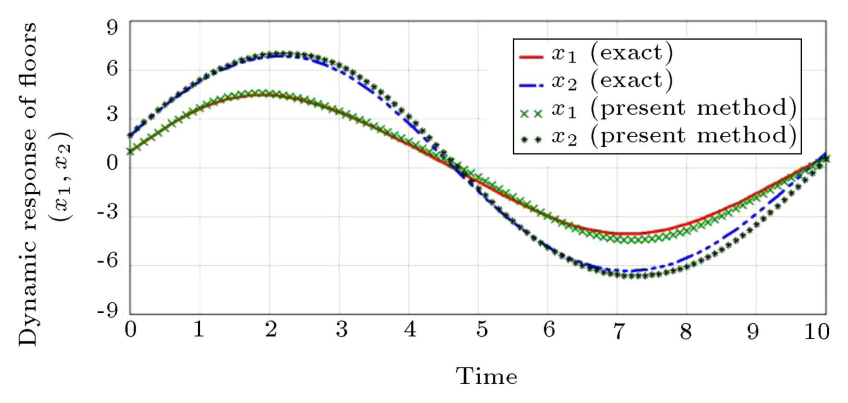

Figure 4. Comparison of the presented method and the exact solution in Example 4.1.

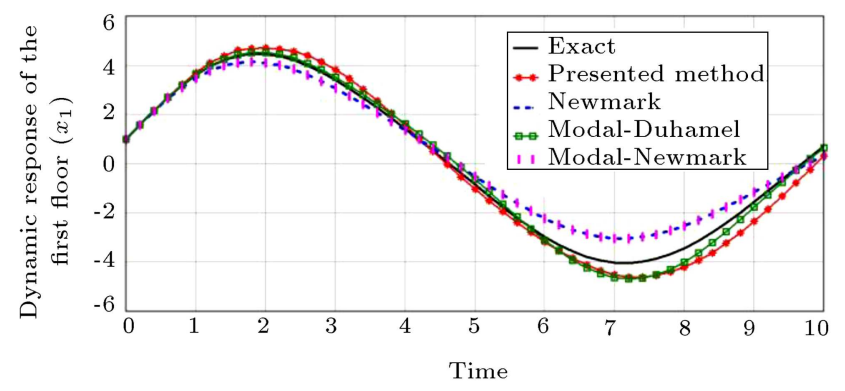

Figure 5. Comparison of the dynamic response of the first floor $\left(x_{1}\right)$ and various methods $(\Delta t=0.2 \mathrm{~s})$.

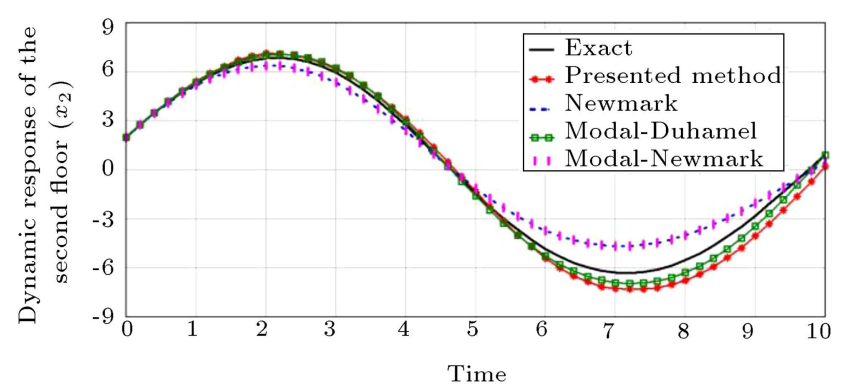

Figure 6. Comparison of the dynamic response of the second floor $\left(x_{2}\right)$ and various methods $(\Delta t=0.2 \mathrm{~s})$.

here, this study compares the accuracy and speed of the analysis of the presented method with those of other conventional methods (such as modal [52], Newmark [23]) and combined techniques (such as modalDuhamel $[53,54]$ and modal-Newmark) (see Figures 5 and 6). Adjustment factors in the Newmark method, which are used to improve the accuracy and stability of the method are respectively selected as follows: $\beta=0.5$ and $\Upsilon=1 / 6$ (typically, these values that yield the linear acceleration method are used in practice). In addition, the combined modal methods will be performed also by converting an n-DOF structure to nSDF systems and applying numerical techniques such as Duhamel and Newmark for the structural analysis. The tolerance of the proposed method is considered to be $10^{-2}$.

From Figures 5 and 6 , it can be observed that the presented method has better and acceptable accuracy than other conventional methods used in the dynamic
Table 4. Required time of analysis in Example 4.1.

\begin{tabular}{lc}
\hline \multicolumn{1}{c}{ Method } & $\begin{array}{c}\text { Required time } \\
\text { for analysis (sec) }\end{array}$ \\
\hline Newmark & 1.511178 \\
Presented Method & 1.915323 \\
Modal-Newmark & 4.845891 \\
Modal-Duhamel & 4.924135 \\
\hline
\end{tabular}

analysis of the MDF structure. In fact, (considering a constant $\Delta t$ ) among all methods, the combined ModalDuhamel and the proposed method have been closer to the exact response to the problem.

Furthermore, in engineering analysis, the time required to calculate the solution, or the speed of numerical technique, is one of the factors influencing the choice of method. Therefore, in this section, in accordance with Table 4, the required times required to conduct the analysis by the various numerical methods are compared. The results show that the methods using the modal techniques are very timeconsuming compared to other methods. For example, the computational time for the proposed approach is shorter than half of the other modal methods.

It must be stated that although the whole damping matrices considered in this study are of a classi$\mathrm{cal} /$ proportional type, it is not generally easy to apply a conventional modal method for non-classical damping, because, in this case, the frequencies, the shape-modes, and damping ratios besides the mass and stiffness matrices, depending on the damping matrix of the system, and the complex modal coordinate must be used (for more details, see [55-57]). On the other hand, it is noteworthy that the energy-based method presented in this research is not subject to any limitations in this regard, and the classical or non-classical damping will be analyzed without a particular modification (it is another advantage of this technique).

Example 4.2. The damped harmonic vibration of a three-story shear building. A two-DOF shear frame is depicted in Figure 7 in which, similar to the previous example, for convenience, the dynamic properties of the structure are selected as: $m_{1}=m_{2}=$ $m_{3}=k_{1}=k_{2}=k_{3}=1, c_{1}=c_{2}=c_{3}=0.1$. In addition, the zero initial conditions are assumed in this example. The structure is subjected to harmonic loads as: $p_{1}=\cos t, p_{2}=\cos 2 t$, and $p_{3}=\cos 3 t$ (all units are compatible).

In this case, the equation of motion is given below:

$$
[m]\{\ddot{x}\}+[c]\{\dot{x}\}+[k]\{x\}=\{p\},
$$

where the mass, damping, and stiffness matrices are: 


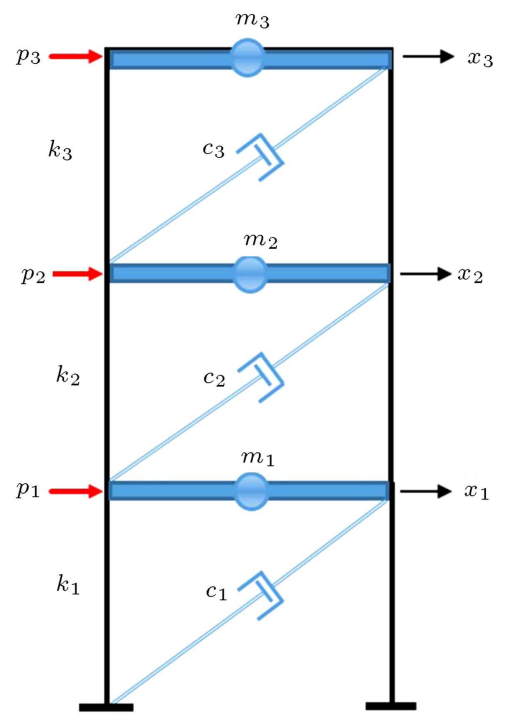

Figure 7. Three-story shear frame under harmonic loads.

$$
\begin{aligned}
& {[m]=\left[\begin{array}{lll}
1 & 0 & 0 \\
0 & 1 & 0 \\
0 & 0 & 1
\end{array}\right], \quad[c]=\left[\begin{array}{ccc}
0.2 & -0.1 & 0 \\
-0.1 & 0.2 & -0.1 \\
0 & -0.1 & 0.1
\end{array}\right],} \\
& {[k]=\left[\begin{array}{ccc}
2 & -1 & 0 \\
-1 & 2 & -1 \\
0 & -1 & 1
\end{array}\right], \quad\{p\}=\left\{\begin{array}{c}
\cos t \\
\cos 2 t \\
\cos 3 t
\end{array}\right\} .}
\end{aligned}
$$

Hence, the governing equations of this problem are given by:

$$
\left\{\begin{array}{l}
\ddot{x}_{1}+0.2 \dot{x}_{1}-0.1 \dot{x}_{2}+2 x_{1}-x_{2}=\cos t \\
\ddot{x}_{2}-0.1 \dot{x}_{1}+0.2 \dot{x}_{2}-0.1 \dot{x}_{3}-x_{1}+2 x_{2}-x_{3}=\cos 2 t \\
\ddot{x}_{3}-0.1 \dot{x}_{2}+0.1 \dot{x}_{3}-x_{2}+x_{3}=\cos 3 t
\end{array}\right.
$$

As for the previous example, the Laplace transform method is used to determine the exact solution of the problem (for details, see Appendix D).

$$
\begin{aligned}
x_{1}= & 0.2131 \exp (-0.01 t) \cos (0.4449 t+0.0330) \\
& -0.9089 \exp (-0.0775 t) \cos (1.2446 t-0.3013) \\
& -0.5188 \exp (-0.1625 t) \cos (1.7946 t+0.5171) \\
& +0.9674 \cos (t-0.2936) \\
& +0.3286 \cos (2 t+0.9803) \\
& +0.002 \cos (3 t+3.9267), \\
x_{2}= & 0.3831 \exp (-0.01 t) \cos (0.4449 t+0.0282) \\
& +0.4042 \exp (-0.0775 t) \cos (1.2446 t+2.8406) \\
& +0.6476 \exp (-0.1625 t) \cos (1.7946 t+0.517)
\end{aligned}
$$

$$
\begin{aligned}
& +0.0962 \cos (t+4.4169) \\
& +0.6581 \cos (2 t+3.7258) \\
& +0.0189 \cos (3 t+0.4542) \\
x_{3}= & 0.4772 \exp (-0.01 t) \cos (0.4449 t+0.0318) \\
& +0.7288 \exp (-0.0775 t) \cos (1.2446 t-0.3012) \\
& +0.4097 \exp (-0.1625 t) \cos (1.7946 t+4.0561) \\
& +0.9618 \cos (t+2.9491) \\
& +0.2235 \cos (2 t+0.8471) \\
& +0.1271 \cos (3 t+3.1911) .
\end{aligned}
$$

\subsection{Applying the energy method}

By applying Eq. (23), the energy balance equations of this system are given as follows:

$$
\begin{aligned}
& 0.5 v_{1}^{2}+\int_{0}^{t}\left[0.2 v_{1}^{2}-0.1 v_{2} v_{1}\right] d t+\int_{0}^{t}\left[2 x_{1}-x_{2}\right] v_{1} d t \\
& =\int_{0}^{t} v_{1} \cos t d t \\
& 0.5 v_{2}^{2}+\int_{0}^{t}\left[0.2 v_{2}^{2}-0.1 v_{1} v_{2}-0.1 v_{3} v_{2}\right] d t \\
& +\int_{0}^{t}\left[2 x_{2}-x_{1}-x_{3}\right] v_{2} d t=\int_{0}^{t} v_{2} \cos 2 t d t \\
& 0.5 v_{3}^{2}+\int_{0}^{t}\left[0.1 v_{3}^{2}-0.1 v_{2} v_{3}\right] d t+\int_{0}^{t}\left[x_{3}-x_{2}\right] v_{3} d t \\
& =\int_{0}^{t} v_{3} \cos 3 t d t .
\end{aligned}
$$

After discretizing Eq. (50) and using Table 2, the same procedure as that in the former example must be performed. In this case, Figures 8 to 10 show the obtained results, where the dynamic response of floors, assuming $\Delta t=0.2 \mathrm{~s}$ and $e=0.01$, is plotted by using the various numerical methods vs. exact solution of the problem.

Figures 8-10 demonstrate that with a fixed size for time intervals, the proposed method in this study together with Modal-Duhamel technique is very close 


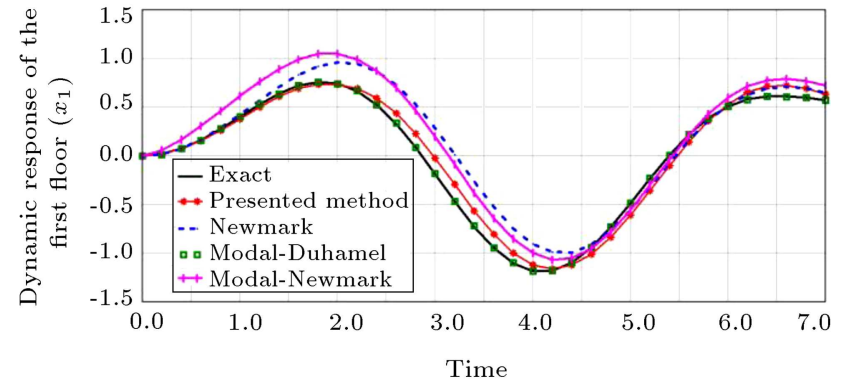

Figure 8. Comparison of the dynamic response of the first floor $\left(x_{1}\right)$ and various methods $(\Delta t=0.2 \mathrm{~s})$.

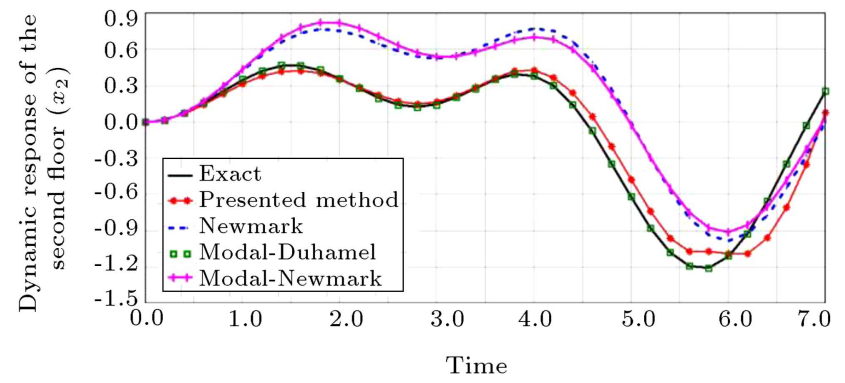

Figure 9. Comparison of the dynamic response of the second floor $\left(x_{2}\right)$ and various methods $(\Delta t=0.2 \mathrm{~s})$.

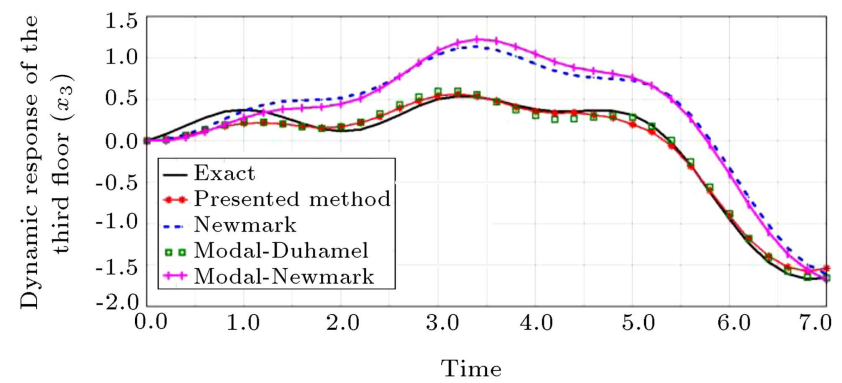

Figure 10. Comparison of the dynamic response of the third floor $\left(x_{3}\right)$ and various methods $(\Delta t=0.2 \mathrm{~s})$.

Table 5. Required time of analysis in Example 4.2.

\begin{tabular}{lc}
\hline \multicolumn{1}{c}{ Method } & $\begin{array}{c}\text { Required time } \\
\text { for analysis (sec) }\end{array}$ \\
\hline Newmark & 1.964039 \\
Presented Method & 2.585024 \\
Modal Duhamel & 4.850705 \\
Modal-Newmark & 4.484707 \\
\hline
\end{tabular}

to the exact solution of the problem, and the methods using Newmark technique (with $\beta=0.5$ and $\Upsilon=1 / 6$ ) do not show appropriate convergence. Here, similar to the previous example, the time required for conducting the analysis of this example is shown in Table 5 , where, similar to the former analysis, the modal techniques are very time consuming compared with others. In addition, note that although the Newmark method

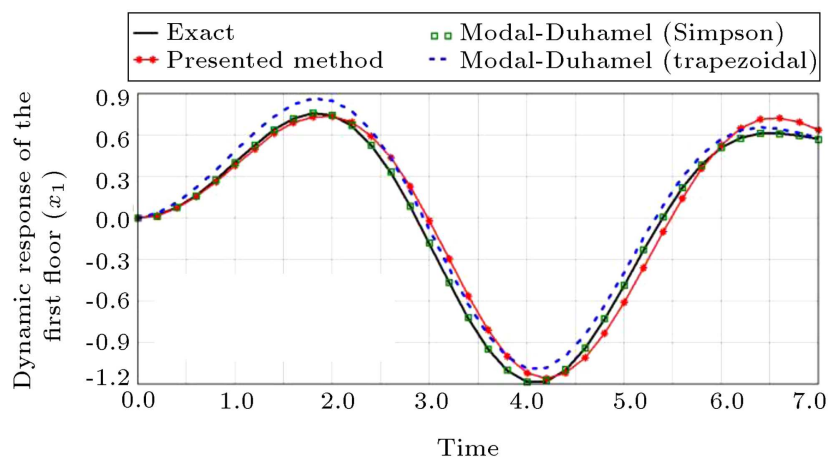

Figure 11. Comparison of the accuracy of the proposed method and two numerical techniques in computing the Duhamel's integral for the first floor.

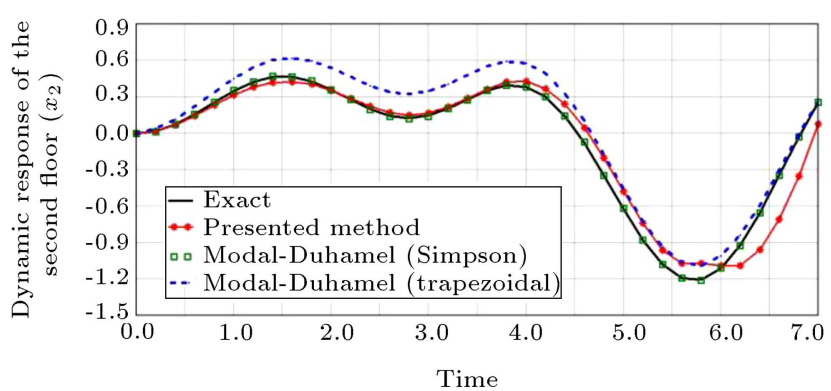

Figure 12. Comparison of the accuracy of the proposed method and two numerical techniques in computing the Duhamel's integral for the second floor.

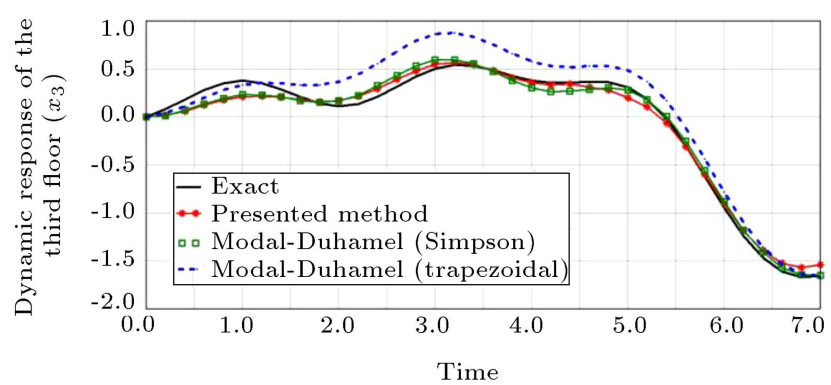

Figure 13. Comparison of the accuracy of the proposed method and two numerical techniques in computing the Duhamel's integral for the third floor.

enjoys a notable speed, its accuracy is not favorable enough in this case compared to other methods.

According to Figures 8-10, the modal-Duhamel method is shown to be more accurate than other approaches. Here, the effect of the numerical technique used in the approximation of the Duhamel integral is investigated. In this regard, in addition to the firstused Simpson rule, the Trapezoidal rule for computing the Duhamel integral is also provided in Figures 1113. Moreover, it must be mentioned that to prevent the cluttered graphs, the results of the Newmark and Modal-Newmark methods are not represented in these figures.

Generally, Figures 11-13 show that the accuracy 


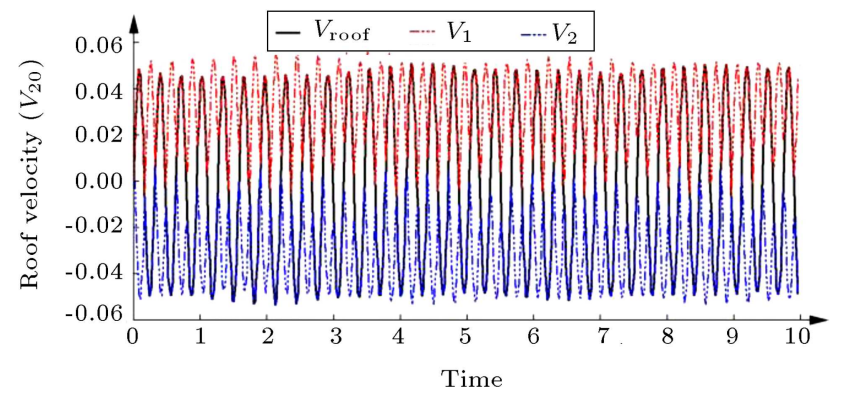

Figure 14. Convergence of velocities in a 20 -story shear-building considering the control point at the roof.

of the Duhamel method is strongly dependent on the numerical method (Simpson with trapezoidal) used in the approximation of this integral. As compared to the proposed method, the application of the Simpson method produces more accurate results and, conversely, the application of the Trapezoidal rule leads to a reduction in the accuracy compared with the presented method.

In the following, to examine the efficiency of the proposed method in the case of large-scale structures, a high-rise 20-story shear frame (as a generalized system of the structure studied in Example 4.2.) is considered with the dynamic properties shown below:

$$
\begin{aligned}
& m_{i}=1, \quad c_{i}=0.1, \quad k_{i}=1, \quad p_{i}=\cos i t \\
& i=1,2, \cdots, 20 .
\end{aligned}
$$

Now, by choosing the last node above the structure (as a control point) and, then, applying the proposed method, this study plots the roof's velocity in 10 seconds versus two converged velocities, i.e., $v_{\text {roof }(1)}$ and $v_{\text {roof (2) }}$, at the end of iterations in the quadratic energy equation, Eq. (24), as displayed in Figure 14.

From Figure 14, it can be seen that, in this largescale system, the roof velocity is properly calculated from the selection of right velocity based on the assumption of continuous velocities in time. Although it appears that future research and studies, especially by considering the nonlinear behavior in other high-rise building systems, are necessary to be done to verify the efficiency of the given method for the general problems of structural dynamics.

Example 4.3. The forced damped vibration of a three-story shear building subjected to an earthquake. Given a three-story shear frame as described in Figure 15 and being subjected to ground motion, EL-Centro earthquake $(P G A=0.3 \mathrm{~g})$ is shown in Figure 15. In addition, the dynamic characteristics of the system are: $m_{1}=m_{2}=m_{3}=1, c_{1}=c_{2}=$ $c_{3}=0.05$, and $k_{1}=k_{2}=k_{3}=10$. Moreover, the zero initial conditions are assumed in this case (all units are compatible).

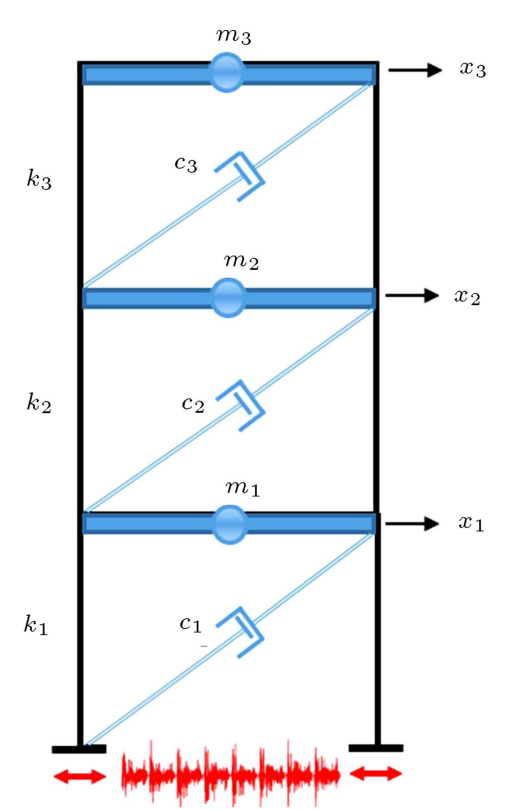

Figure 15. Three-story shear building under earthquake loading.

In this case, due to earthquake loading, by the definition of effective force $\left(p_{\text {eff }}\right)$, the equation of motion is given below:

$$
[m]\{\ddot{x}\}+[c]\{\dot{x}\}+[k]\{x\}=\left\{p_{e f f}\right\},
$$

where $p_{\text {eff }}$ denotes the negative product of the mass matrix $[m],\{l\}$ is the influencing coefficient vector, and $\left\{\ddot{x}_{g}\right\}$ is the acceleration vector of ground motion, as given in the following relation:

$$
\left\{p_{\text {eff }}\right\}=-[m]\{l\}\left\{\ddot{x}_{g}\right\} .
$$

For the problem at hand, the influencing coefficient vector is:

$$
\{l\}^{T}=\{1,1,1\},
$$

where mass, damping, and stiffness matrices are given below:

$$
\begin{aligned}
& {[m]=\left[\begin{array}{lll}
1 & 0 & 0 \\
0 & 1 & 0 \\
0 & 0 & 1
\end{array}\right],} \\
& {\left[c=\left[\begin{array}{ccc}
0.1 & -0.05 & 0 \\
-0.05 & 0.1 & -0.05 \\
0 & -0.05 & 0.05
\end{array}\right],\right.} \\
& {[k]=\left[\begin{array}{ccc}
20 & -10 & 0 \\
-10 & 20 & -10 \\
0 & -10 & 10
\end{array}\right] .}
\end{aligned}
$$

\subsection{Applying the energy method}

According to Table 2 and the two examples mentioned 


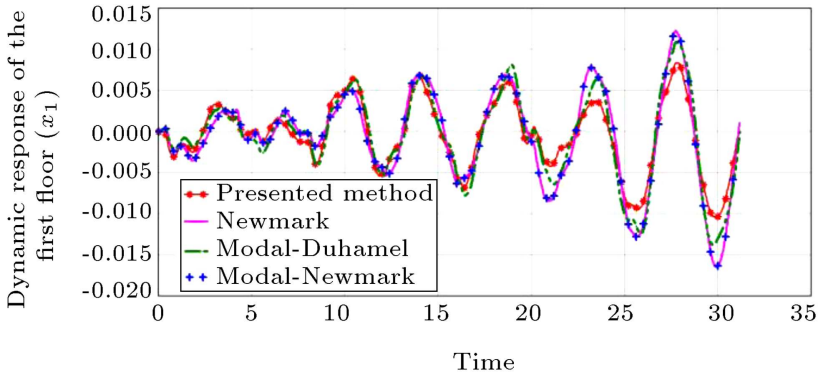

Figure 16. Comparison of the dynamic response of the first floor $\left(x_{1}\right)$ and various methods $(\Delta t=0.2 \mathrm{~s})$.

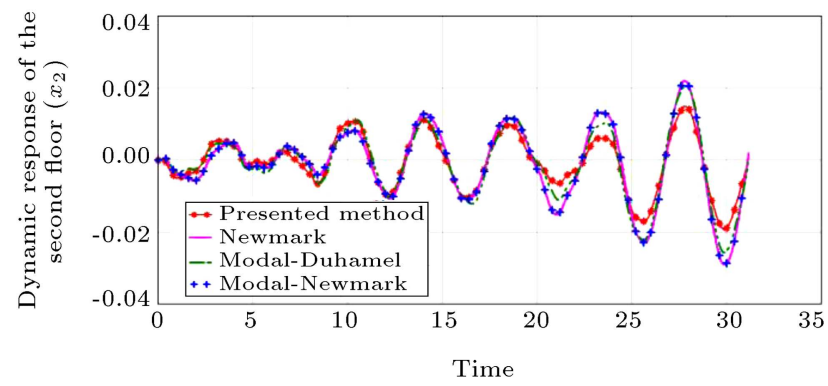

Figure 17. Comparison of the dynamic response of the second floor $\left(x_{2}\right)$ and various methods $(\Delta t=0.2 \mathrm{~s})$.

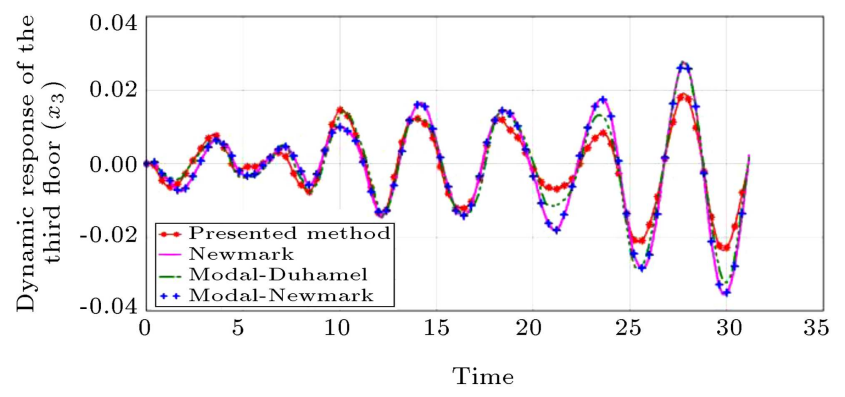

Figure 18. Comparison of the dynamic response of the third floor $\left(x_{3}\right)$ and various methods $(\Delta t=0.2 \mathrm{~s})$.

earlier, by applying the energy method and assuming previous assumptions, except the value of tolerance being equal to $e=10^{-4}$, the dynamic response of the structure can be plotted. As is clear, in this kind of problem, there is no closed-form analytical solution that can compare the results. Thus, only the results of various numerical methods (at a fixed time interval equal to 0.02) are plotted in Figures 16 to 18.

Please note that the marked points in Figures 1618 do not denote the time steps and are merely selected to make a distinction between the results. In addition, the obtained results of Newmark and Modal-Newmark methods overlap and cannot properly be identified. According to the figures, acceptable agreement between the presented method and other methods can be seen. Hence, this method can be used for performing the long-time dynamic analysis of shear frames such as seismic analyses.
Table 6. Required time of analysis in Example 4.3.

\begin{tabular}{lc}
\hline \multicolumn{1}{c}{ Method } & $\begin{array}{c}\text { Required time } \\
\text { for analysis (sec) }\end{array}$ \\
\hline Newmark & 18.405511 \\
Presented method & 20.076837 \\
Modal-Newmark & 25.035491 \\
Modal-Duhamel & 28.462937 \\
\hline
\end{tabular}

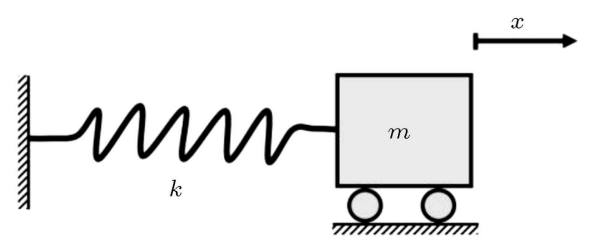

Figure 19. A Single-Degree-of-Freedom (SDF) system for accuracy and stability analysis of numerical analyses.

Once again, to compare the speed of analyses, Table 6 shows the time required to analyze the third example of this investigation. Similar to the previous examples, it can be observed that, at a constant time interval, the proposed method regarding computational time is ranked second after Newmark method.

At the first glance, although the times (durations) given in Table 6 may look great for a small 3-DOF structure, it should be mentioned here that these times should include the execution time of all the commands written within the MATLAB program (e.g., timeconsuming syntaxes like (xlsread)). In other words, these values do not indicate the real time of the implementation of the integration schemes and are used only for making a comparison between different types of methods.

\subsection{Stability and accuracy analysis}

Here, the effects of time step size on the accuracy and stability of the presented method are discussed. In this regard, Bathe [58] proposed a technique based on the free response analysis of a simple SDF system, as shown in Figure 19. For simplicity, the following parameters in a compatible unit system are assumed as follows: $m=1, k=4 \pi^{2}, x_{0}=0$, and $v_{0}=1$. The free response of this system (exact solution of the problem) can be written as follows:

$$
x(t)=(\sin 2 \pi t) / 2 \pi .
$$

With respect to this exact response, the values for the period and amplitude of the vibrational motion are equal to $T_{\text {Exact }}=1$ and $A_{\text {Exact }}=1 / 2 \pi$ respectively. Obviously, the numerical solution obtained from the presented method will differ from these values. Therefore, it would be appropriate to define the two following parameters:

$$
R_{T}=\left|\left(T_{\text {Exact }}-T_{\text {Num }}\right) / T_{\text {Exact }}\right|,
$$




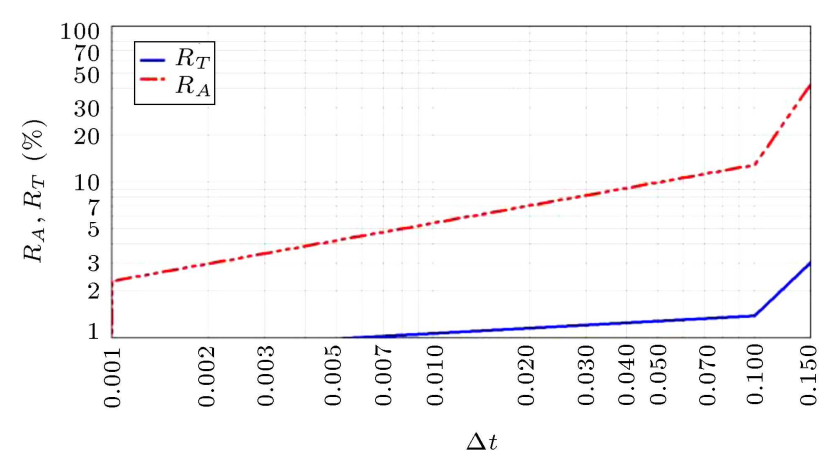

Figure 20. The effects of the different time step size on the accuracy and stability of the presented method.

and:

$$
R_{A}=\left|\left(A_{\text {Exact }}-A_{N u m}\right) / A_{\text {Exact }}\right|,
$$

where $R_{T}$ and $R_{A}$ represent the numerical error in period and amplitude characteristics of the vibrational system, respectively. $T_{N u m l}$ and $A_{N u m}$ also are the period and amplitude obtained from the numerical method, which are the functions of the size of the time step, $\Delta t$, used to discretize time. Thus, considering different values of the time step, one can plot the parameters $R_{T}$ and $R_{A}$, as shown in Figure 20 .

According to Figure 20, as the time step increases (with an increase in the numerical error in the system response), the accuracy of the solution reduces, as expected. For example, when the time step size is $\Delta t=0.1$, the relative errors in the period and amplitude of the system are equal to about $1.5 \%$ and $13 \%$, respectively. In general, the results of this section show greater sensitivity to the amplitude of motion than the period (this is in line with the results of [27]).

Moreover, based on a closer observation of this figure, by increasing the value of time step, numerical errors increase significantly at a certain value (about 0.1 to 0.15 ), indicating that instability occurs in the numerical solution. For example, in the case of $\Delta t=$ 0.15 , the use of about 6-7 points for the approximation of a complete sine wave led to a significant error. Therefore, selecting an appropriate value of $\Delta t$ is essential in practice, because such a larger value can cause instability by eliminating the precision of solution. On the other hand, small $\Delta t$ also increases the computational time. Consequently, an optimum size for time step should be used in practical dynamic analyses.

\section{Conclusions}

In this paper, a novel step-by-step solution technique based on the energy method was presented for the dynamic analysis of shear frames as one of the applicable structures in practice. Rather than working with the equation of motions, this method solved the energy balance relationships, as characterized by some advantages such as the reduction of unknowns. The proposed method for analyzing various examples including harmonic and earthquake loading was presented and performed. The main implications of the study can be listed as follows:

- The proposed method enjoys higher accuracy than other common methods (e.g., it is more accurate than Newmark method);

- Compared to other time integration methods such as Newmark, the proposed method gives a chance to avoid the necessity of selecting and calibrating the velocity and acceleration adjustment parameters $\gamma$, $\beta$;

- Modal methods, which have shown good accuracy in combination with Duhamel's Integral, has complex mathematic relationships, particularly with increasing the degrees of freedom of the structure; in addition, as observed in this study, they are more time consuming than other techniques;

- The presented method, with a simple mathematical algorithm, has good accuracy and speed of analysis. By setting an allowable tolerance threshold (usually in the range of 0.01-0.0001), the method can be used in practical dynamic analyses of shear frames.

Finally, it should be noted that the ideas expressed in this research have the capability to be applied to other engineering structures and also non-linear systems with some modifications.

\section{References}

1. Wilson, E.L. "Dynamic analysis", In ThreeDimensional Static and Dynamic Analysis of Structures, 3rd Edn., pp. 165-175, Computers and Structures, Berkeley, California, USA (2002).

2. Craig, R. and Kurila, A. "Preface to structural dynamics", In Fundamentals of Structural Dynamics, 2nd Edn., pp. 13-14, Wiley, New Jersey, USA (2006).

3. Clough, R. and Penzien, J. "Earthquake engineering", In Dynamics of Structures, 3rd Edn., pp. 555-730, Computers and Structures, Berkeley, California, USA (2013).

4. Chopra, A.K. "Earthquake analysis of arch dams: factors to be considered", ASCE, 138(2), pp. 205-214 (2012).

5. Chopra, A.K. "Earthquake analysis of concrete dams: factors to be considered", 10th U.S. National Conference on Earthquake Engineering, Anchorage, Alaska, USA (2014).

6. Feng, M.Q., Fukuda, Y., Feng, D., and Mizuta, M. "Nontarget vision sensor for remote measurement of bridge dynamic response", J. Bridg. Eng., 20(2015), pp. 1-12 (2015). 
7. Krodkiewski, J.M., Mechanical Vibration, Melbourne University, pp. 1-247, Melbourne, Australia (2008).

8. Chopra, A.K. "Single degree-of-freedom systems", In Dynamics of Structures, 4th Edn., pp. 1-307, Prentice Hall, Berkeley, University of California, USA (2013).

9. Makem, J.E., Armstrong, C.G., and Robinson, T.T. "Automatic decomposition and efficient semistructured meshing of complex solids", Engineering with Computers, 30(3), pp. 345-361 (2014).

10. Kougioumtzoglou, I.A. and Spanos P.D. "Nonlinear MDOF system stochastic response determination via a dimension reduction approach", Comput. Struct., 126(1), pp. 135-148 (2013).

11. Brahmi, K., Bouhaddi, N., and Fillod, R., Reduction of Junction Degree of Freedom in Certain Methods of Dynamic Substructure Synthesis, The International Society for Optical Engineering, Spie International Society for Optical, Bellingham, USA, pp. 1763-1763 (1995).

12. Kreyszig, E. "Numerical analysis", In Advanced Engineering Mathematics, 10th Edn., Wiley, pp. 787-949, New York, USA (2005).

13. Blahut, E.R. "Fast algorithms for the discrete Fourier transform", In Fast Algorithms for Signal Processing, pp. 68-114, Cambridge University Press, New York, USA (2010).

14. Elizalde-Siller, H.R. "Non-linear modal analysis methods for engineering structures", Doctoral dissertation, Imperial College London, UK (2004).

15. Wong, K.K.F. "Nonlinear dynamic analysis of structures using modal superposition", ASCE Structures Congress 2011, pp. 770-781, Las Vegas, Nevada, USA (2011).

16. Dou, S. and Jakob, S.J. "Optimization of hardening/softening behavior of plane frame structures using nonlinear normal modes", Comput. Struct., 164, pp. 63-74 (2016).

17. Dou, S. "Gradient-based optimization in nonlinear structural dynamics", PhD Thesis, Mechanical Engineering, DTU, Denmark (2015).

18. Dou, S. and Jakob, S.J. "Analytical sensitivity analysis and topology optimization of nonlinear resonant structures with hardening and softening behavior", 17th U.S. National Congress on Theoretical and Applied Mechanics, East Lansing, Michigan, USA, pp. 1-3 (2014).

19. Chopra, A.K. and Goel, R.K. "A modal pushover analysis procedure to estimate seismic demands for unsymmetric-plan buildings", Earthq. Eng. Struct. Dyn., 33(8), pp. 903-927 (2004).
20. Chopra, A.K. and Goel, R.K. "A modal pushover analysis procedure for estimating seismic demands for buildings", Earthquake Engineering and Structural Dynamics, 31(3), pp. 561-582 (2002).

21. Cheng, F.Y. "Eigensolution techniques and undamped response analysis of multiple-degree-of-freedom systems", In Matrix Analysis of Structural Dynamics, pp. 48-98, Marcel Dekker, Rolla, Missouri, USA (2001).

22. Bathe, K.J. "Eigen problems", In Finite Element Procedures in Engineering Analysis, pp. 838-979, Prentice Hall, New Jersey, USA (1996).

23. Newmark, N.M. "A method of computation for structural dynamics", Journal of the Engineering Mechanics, 85(7), pp. 67-94 (1959).

24. Houbolt, J.C. "A recurrence matrix solution for the dynamic response of elastic aircraft", J. Aeronaut. Sci., 17(9), pp. 540-550 (1950).

25. Wilson, E.L., Farhoomand, I., and Bathe, K.J. "Nonlinear dynamic analysis of complex structures", Earthq. Eng. Struct. Dyn., 1(March 1972), pp. 241252 (1973).

26. Bathe, K.J. and Wilson, E.L., Numerical Methods in Finite Element Analysis, pp. 1-528, Prentice Hall, New Jersey, USA (1976).

27. Bathe, K.J. and Wilson, E.L. "Stability and accuracy analysis of direct integration methods", Earthq. Eng. Struct. Dyn., 1(3), pp. 283-291 (1972).

28. Felippa, C.A. and Park, K.C. "Direct time integration method in nonlinear structural dynamics", Comput. Method Appl. Mech. Eng., 17(18), pp. 277-313 (1979).

29. Johnson, D.E. "A proof of the stability of the houbolt method", AIA A, 4(8), pp. 1450-1451 (1966).

30. Gladwell, I. and Thomas, R. "Stability properties of the Newmark, Houbolt and Wilson methods", Int. J. Numer. Methods Anal. Methods Eng., 4(August 1979), pp. 143-158 (1980).

31. Park, K.C. "An improved stiffly stable method for direct integration of nonlinear structural dynamic equations", J. Appl. Mech., 42(2), pp. 464-470 (1975).

32. Bathe, K.J. and Wilson, E.L. "NONSAP - A nonlinear structural analysis program", Nucl. Eng. Des., 29(2), pp. 266-293 (1974).

33. Paultre, P. "Direct time integration of linear systems", In Dynamics of Structures, pp. 223-248, John Wiley \& Sons, Surrey, GBR (2013).

34. Acary, V. "Energy conservation and dissipation properties of time-integration methods for nonsmooth elastodynamics with contact", Journal of Applied Mathematics and Mechanics, 96(5), pp. 585-603 (2016). 
35. Fleming, A., Penesis, I., Macfarlane, G., Bose, N., and Denniss, T. "Energy balance analysis for an oscillating water column wave energy converter", Ocean Engineering, 54, pp. 26-33 (2012).

36. Lee, T., Leok, M., and McClamroch, N.H. "Geometric numerical integration for complex dynamics of tethered spacecraft", American Control Conference (ACC), San Francisco, California, USA, pp. 1885-1891 (2011).

37. Dowell, E.H. "Nonlinear aeroelasticity", In A Modern Course in Aeroelasticity, pp. 487-529, Springer, NC, USA (2015).

38. Stanton, S.C., Erturk, A., Mann, B.P., Dowell, E.H., and Inman, D.J. "Nonlinear nonconservative behavior and modeling of piezoelectric energy harvesters including proof mass effects", J. Intell. Mater. Syst. Struct., 23(2), pp. 183-199 (2012).

39. Hirsch, C. "An Initial Guide to CFD", In Numerical Computation of Internal and External Flows: The Fundamentals of Computational Fluid Dynamics, 2nd edition, pp. 1-12, Elsevier, Amesterdam, Netherlands (2007).

40. Yazdi, M.K. and Tehrani, P.H. "The energy balance to nonlinear oscillations via Jacobi collocation method", Alexandria Eng. J., 54(2), pp. 99-103 (2015).

41. Mehdipour, I., Ganji, D.D., and Mozaffari, M. "Application of the energy balance method to nonlinear vibrating equations", Current Applied Physics, 10(1), pp. 104-112 (2010).

42. Jalili Sadr Abad, M., Mahmoudi, M., and Dowell, E.H. "Dynamic analysis of SDOF systems using modified energy method", ASIAN J. Civ. Eng., 18(7), pp. 11251146 (2017).

43. Paz, M. and Leigh, W. "Structures modeled as shear buildings", In Structural Dynamics, pp. 205-231, Springer, Boston, USA (2004).

44. Feynman, R.P., Leighton, R.B., and Sands, M., The Feynman Lectures on Physics, Desktop Edition, 1, Basic Books (2013).

45. Hall, K.C., Ekici, K., Thomas, J.P., and Dowell, E.H. "Harmonic balance methods applied to computational fluid dynamics problems", Int. J. Comut. Fluid Dyn., $\mathbf{2 7}(2)$, pp. 52-67 (2013).

46. Light, J.C. and Walker, R.B. "An R matrix approach to the solution of coupled equations for atom-molecule reactive scattering", J. Chem. Phys., 65(10), pp. 42724282 (1976).

47. Wang, Y., Ding, H., and Chen, L.Q. "Asymptotic solutions of coupled equations of supercritically axially moving beam", Nonlinear Dyn., 87(1), pp. 25-36 (2016).
48. Armour, E.A.G. and Plummer, M. "Calculation of the resonant contribution to Zeff(k0) using close-coupled equations for positron-molecule scattering", J. Phys. B At. Mol. Opt. Phys., 49(15), pp. 1-11 (2016).

49. Haojiang, D. "General solutions for coupled equations for piezoelectric media", Int. J. Solids Struct., 33(16), pp. 2283-2298 (1996).

50. Polyanin, A.D. and Lychev, S.A. "Decomposition methods for coupled 3D equations of applied mathematics and continuum mechanics: Partial survey, classification, new results, and generalizations", Appl. Math. Model., 40(4), pp. 3298-3324 (2016).

51. Mourad, A. and Kamel, Z. "An efficient method for solving the MAS stiff system of nonlinearly coupled equations: Application to the pseudoelastic response of shape memory alloys (SMA)", IOP Conf. Ser. Mater. Sci. Eng., 123(1), Miskolc-Lillafüred, Hungary, pp. 15 (2016).

52. Nikitas, N., Macdonald, J.H.G., and Tsavdaridis, K.D. "Modal analysis", Encycl. Earthq. Eng., 23, pp. 1-22 (2014).

53. Van Putten, M.H., Introduction to Methods of Approximation in Physics and Astronomy, Springer, Singapore (2017).

54. Duhamel, J.M.C., Elements de Calcul Infinitesimal, Mallet-Bachelier, France (1860) (In French).

55. Gavin, H.P., Classical Damping, Non-classical Damping, and Complex Modes, Dep. Civ. Environ. Eng. Duke Univ. NC, USA (2016).

56. Ghahari, S.F., Abazarsa, F., and Taciroglu, E. "Blind modal identification of non-classically damped structures under non-stationary excitations", Struct. Control Heal. Monit., 24(6), pp. 987-1006 (2017).

57. Cruz, C. and Miranda, E. "Evaluation of the Rayleigh damping model for buildings", Eng. Struct, 138, pp. 324-336 (2017).

58. Bathe, K.J. "Solution of equilibrium equations in dynamic analysis", In Finite Element Procedures, 2nd Edn., pp. 768-837, Prentice Hall, New Jersey, USA (2014).

59. Ben-Israel, A. "A Newton-Raphson method for the solution of systems of equations", J. Math. Anal. Its Appl., 15, pp. 243-252 (1966).

60. Rheinboldt, W.C. "Methods of Newton Type", In Methods for Solving Systems of Nonlinear Equations, 2nd Edn., pp. 35-43, Society for Industrial and Applied Mathematics (SIAM), Pittsburg, Pennsylvania, USA (1998). 
Appendix A. Discretization of integral energy equations

\section{A.1. Trapezoidal rule}

The value of $\int_{0}^{\Delta t} f(t) d t$ can be evaluated by the Trapezoidal rule:

$$
\int_{0}^{\Delta t} f(t) d t \simeq \frac{\Delta t}{2}[f(0)+f(\Delta t)] .
$$

Recall Eq. (23):

$$
\begin{aligned}
\frac{1}{2} m_{i} v_{i}^{2} & -\frac{1}{2} m_{i} v_{i(0)}^{2}+\int_{0}^{t}\left[\left(c_{i}+c_{i+1}\right) v_{i}^{2}-c_{i} v_{i-1} v_{i}\right. \\
\left.-c_{i+1} v_{i+1} v_{i}\right] d t+ & \int_{0}^{t}\left[\left(k_{i}+k_{i+1}\right) x_{i}-k_{i} x_{i-1}\right. \\
\left.-k_{i+1} x_{i+1}\right] v_{i} d t & =\int_{0}^{t} p_{i} v_{i} d t .
\end{aligned}
$$

Now, considering Eq. (23) and based on the trapezoidal rule, the integrals in this expression can be discretized by Eqs. (A.2)-(A.4) as shown in Box I.

Substituting Eqs. (A.2)-(A.4) into Eq. (23) and rearranging with regard to velocities yields the following:

$$
\begin{aligned}
A_{i} v_{i(\Delta t)}^{2} & +B_{i} v_{i-1(\Delta t)} v_{i(\Delta t)}+C_{i} v_{i+1(\Delta t)} v_{i(\Delta t)} \\
& +D_{i} v_{i(\Delta t)}+E_{i}=0
\end{aligned}
$$

where:

$$
\begin{aligned}
A_{i}= & 0.5 m_{i}+0.5 \Delta t\left(c_{i}+c_{i+1}\right) \\
B_{i}= & -0.5 \Delta t . c_{i}, \quad C_{i}=-0.5 \Delta t . c_{i+1}, \\
D_{i}= & 0.5 \Delta t\left[\left(k_{i}+k_{i+1}\right) x_{i(\Delta t)}-k_{i} \cdot x_{i-1(\Delta t)}\right. \\
& \left.-k_{i+1} \cdot x_{i+1(\Delta t)}-p_{i(\Delta t)}\right] \\
E_{i}= & -0.5 m_{i} v_{i(0)}^{2}+0.5 \Delta t . v_{i(0)}\left[\left(c_{i}+c_{i+1}\right) v_{i(0)}\right. \\
& -c_{i} \cdot v_{i-1(0)}+c_{i+1} \cdot v_{i+1(0)}+\left(k_{i}+k_{i+1}\right) x_{i(0)} \\
& \left.-k_{i} x_{i-1(0)}-k_{i+1} x_{i+1(0)}-p_{i(0)}\right] .
\end{aligned}
$$

\section{A.2. Simpson rule}

Consider the $j$ th time step, i.e., $t=j \Delta t(j=2,3, \cdots)$. In this case, $\int_{0}^{t} f(t) d t$ can be approximated by the composite Simpson rule as follows:

$$
\begin{aligned}
& \int_{0}^{\Delta t} \underbrace{\left[\left(c_{i}+c_{i+1}\right) v_{i}^{2}(t)-c_{i} v_{i-1}(t) v_{i}(t)-c_{i+1} v_{i+1}(t) v_{i}(t)\right]}_{f(t)} d t=\frac{\Delta t}{2}\{\underbrace{\left[\left(c_{i}+c_{i+1}\right) v_{i(0)}^{2}-c_{i} v_{i-1(0)} v_{i(0)}-c_{i+1} v_{i+1(0)} v_{i(0)}\right]}_{f(0)} \\
& +\underbrace{\left[\left(c_{i}+c_{i+1}\right) v_{i(\Delta t)}^{2}-c_{i} v_{i-1(\Delta t)} v_{i(\Delta t)}-c_{i+1} v_{i+1(\Delta t)} v_{i(\Delta t)}\right]}_{f(\Delta t)}\} \\
& \int_{0}^{\Delta t} \underbrace{\left[\left(k_{i}+k_{i+1}\right) x_{i}(t)-k_{i} x_{i-1}(t)-k_{i+1} x_{i+1}(t)\right] v_{i}(t)}_{f(t)} d t=\frac{\Delta t}{2}\{\underbrace{\left[\left(k_{i}+k_{i+1}\right) x_{i(0)}-k_{i} x_{i-1(0)}-k_{i+1} x_{i+1(0)}\right] v_{i(0)}}_{f(0)} \\
& +\underbrace{\left[\left(k_{i}+k_{i+1}\right) x_{i(\Delta t)}-k_{i} x_{i-1(\Delta t)}-k_{i+1} x_{i+1(\Delta t)}\right] v_{i(\Delta t)}}_{f(\Delta t)}\}, \\
& \int_{0}^{\Delta t} \underbrace{\left[p_{i}(t) v_{i}(t)\right]}_{f(t)} d t=\frac{\Delta t}{2}\{\underbrace{p_{i(0)} v_{i(0)}}_{f(0)}+\underbrace{p_{i(\Delta t)} v_{i(\Delta t)}}_{f(\Delta t)}\} .
\end{aligned}
$$




$$
\begin{aligned}
\int_{0}^{j \Delta t} f(t) d t \simeq & \frac{\Delta t}{6}\left[f_{(0)}+4 f_{(\Delta t)}+4 f_{(2 \Delta t)}+\cdots\right. \\
& \left.+4 f_{((j-1) \Delta t)}+f_{(j \Delta t)}\right] .
\end{aligned}
$$

Similar to the previous case, the discretized form of Eq. (23) using Simpson rule is given by Eqs. (A.6)-(A.8) as shown in Box II. Hence, by inserting Eqs. (A.6)(A.8) in Eq. (23) and simplifying them, one can obtain the discretized form of energy equations.

$$
\begin{aligned}
A_{i} v_{i(\Delta t)}^{2} & +B_{i} v_{i-1(\Delta t)} v_{i(\Delta t)}+C_{i} v_{i+1(\Delta t)} v_{i(\Delta t)} \\
& +D_{i} v_{i(\Delta t)}+E_{i}=0
\end{aligned}
$$

where:

$$
\begin{aligned}
& A_{i}=0.5 m_{i}+(\Delta t / 3)\left(c_{i}+c_{i+1}\right), \\
& B_{i}=-(\Delta t / 3) \cdot c_{i}, \quad C_{i}=-(\Delta t / 3) \cdot c_{i+1},
\end{aligned}
$$

$$
\begin{aligned}
& D_{i}=(\Delta t / 3)\left[\left(k_{i}+k_{i+1}\right) x_{i(j \Delta t)}-k_{i} \cdot x_{i-1(j \Delta t)}\right. \\
& \left.-k_{i+1} \cdot x_{i+1(j \Delta t)}-p_{i(j \Delta t)}\right], \\
& E_{i}=-0.5 m_{i} v_{i(0)}^{2}+(\Delta t / 3)\left\{v _ { i ( 0 ) } \left[\left(c_{i}+c_{i+1}\right) v_{i(0)}\right.\right. \\
& -c_{i} \cdot v_{i-1(0)}+c_{i+1} \cdot v_{i+1(0)}+\left(k_{i}+k_{i+1}\right) x_{i(0)} \\
& \left.-k_{i} x_{i-1(0)}-k_{i+1} x_{i+1(0)}-p_{i(0)}\right] \\
& +4 v_{i(\Delta t)}\left[\left(c_{i}+c_{i+1}\right) v_{i(\Delta t)}-c_{i} \cdot v_{i-1(\Delta t)}\right. \\
& +c_{i+1} \cdot v_{i+1(\Delta t)}+\left(k_{i}+k_{i+1}\right) x_{i(\Delta t)} \\
& -k_{i} x_{i-1(\Delta t)}-k_{i+1} x_{i+1(\Delta t)} \\
& \left.-p_{i(\Delta t)}\right]+2 v_{i(2 \Delta t)}\left[\left(c_{i}+c_{i+1}\right) v_{i(2 \Delta t)}\right. \\
& -c_{i} \cdot v_{i-1(2 \Delta t)}+c_{i+1} \cdot v_{i+1(2 \Delta t)} \\
& +\left(k_{i}+k_{i+1}\right) x_{i(2 \Delta t)}-k_{i} x_{i-1(2 \Delta t)}
\end{aligned}
$$

$$
\begin{aligned}
& \int_{0}^{j \Delta t} \underbrace{\left[\left(c_{i}+c_{i+1}\right) v_{i}^{2}(t)-c_{i} v_{i-1}(t) v_{i}(t)-c_{i+1} v_{i+1}(t) v_{i}(t)\right]}_{f(t)} d t=\frac{\Delta t}{6}\{\underbrace{\left[\left(c_{i}+c_{i+1}\right) v_{i(0)}^{2}-c_{i} v_{i-1(0)} v_{i(0)}-c_{i+1} v_{i+1(0)} v_{i(0)}\right]}_{f(0)} \\
& +4 \underbrace{\left[\left(c_{i}+c_{i+1}\right) v_{i(\Delta t)}^{2}-c_{i} v_{i-1(\Delta t)} v_{i(\Delta t)}-c_{i+1} v_{i+1(\Delta t)} v_{i(\Delta t)}\right]}_{f(\Delta t)}+\cdots \\
& +\underbrace{\left[\left(c_{i}+c_{i+1}\right) v_{i(j \Delta t)}^{2}-c_{i} v_{i-1(j \Delta t)} v_{i(j \Delta t)}-c_{i+1} v_{i+1(j \Delta t)} v_{i(j \Delta t)}\right]}_{f(j \Delta t)}\} \\
& \int_{0}^{j \Delta t} \underbrace{\left[\left(k_{i}+k_{i+1}\right) x_{i}(t)-k_{i} x_{i-1}(t)-k_{i+1} x_{i+1}(t)\right] v_{i}(t)}_{f(t)} d t=\frac{\Delta t}{6}\{\underbrace{\left[\left(k_{i}+k_{i+1}\right) x_{i(0)}-k_{i} x_{i-1(0)}-k_{i+1} x_{i+1(0)}\right] v_{i(0)}}_{f(0)} \\
& +4 \underbrace{\left[\left(k_{i}+k_{i+1}\right) x_{i(\Delta t)}-k_{i} x_{i-1(\Delta t)}-k_{i+1} x_{i+1(\Delta t)}\right] v_{i(\Delta t)}}_{f(\Delta t)}+\cdots \\
& +\underbrace{\left[\left(k_{i}+k_{i+1}\right) x_{i(j \Delta t)}-k_{i} x_{i-1(j \Delta t)}-k_{i+1} x_{i+1(j \Delta t)}\right] v_{i(j \Delta t)}}_{f(j \Delta t)}\}, \\
& \int_{0}^{\Delta t} \underbrace{\left[p_{i}(t) v_{i}(t)\right]}_{f(t)} d t=\frac{\Delta t}{6}\{\underbrace{p_{i(0)} v_{i(0)}}_{f(0)}+4 \underbrace{p_{i(\Delta t)} v_{i(\Delta t)}}_{f(\Delta t)}+\cdots+\underbrace{p_{i(j \Delta t)} v_{i(j \Delta t)}}_{f(j \Delta t)}\} .
\end{aligned}
$$




$$
\begin{aligned}
& \left.-k_{i+1} x_{i+1(2 \Delta t)}-p_{i(2 \Delta t)}\right]+\cdots \\
& +v_{i(j \Delta t)}\left[\left(c_{i}+c_{i+1}\right) v_{i(j \Delta t)}-c_{i} \cdot v_{i-1(j \Delta t)}\right. \\
& +c_{i+1} \cdot v_{i+1(j \Delta t)}+\left(k_{i}+k_{i+1}\right) x_{i(j \Delta t)} \\
& \left.\left.-k_{i} x_{i-1(j \Delta t)}-k_{i+1} x_{i+1(j \Delta t)}-p_{i(j \Delta t)}\right]\right\} .
\end{aligned}
$$

(26 rep.)

\section{Appendix B. The Newton-Raphson method for solving Eq. (37)}

Two functions $\left(f_{1}, f_{2}\right)$ for each equation in Eq. (37) are defined as follows:

$$
\begin{aligned}
& f_{1}=0.511 v_{1}^{2}-0.008 v_{2} v_{1}+0.01 v_{1}-4.497=0 \\
& f_{2}=0.508 v_{2}^{2}-0.008 v_{2} v_{1}+0.055 v_{2}-7.768=0 .
\end{aligned}
$$

Then, by extending the Newton-Raphson method to the system of equations, to find the root of $f_{1}$ and $f_{2}$, one may write the following sequence [59]:

$$
\{\mathbf{v}\}_{i+1}=\{\mathbf{v}\}_{i}-[\mathbf{J}]_{i}^{-1}\{\mathbf{f}\}_{i} \quad i=0,1,2, \cdots .
$$

Here, the subscript $i$ represents the iteration number to achieve the convergence criterion; $\{\mathbf{v}\}$ is the vector of unknowns (velocities); $\{\mathbf{f}\}$ is the vector of functions; $[\mathbf{J}]$ denotes the Jacobian matrix.

$$
\{\mathbf{v}\}=\left\{\begin{array}{l}
v_{1} \\
v_{2}
\end{array}\right\}, \quad\{\mathbf{f}\}=\left\{\begin{array}{l}
f_{1} \\
f_{2}
\end{array}\right\}, \quad[\mathbf{J}]=\left[\begin{array}{ll}
\frac{\partial f_{1}}{\partial v_{1}} & \frac{\partial f_{1}}{\partial v_{2}} \\
\frac{\partial f_{2}}{\partial v_{1}} & \frac{\partial f_{2}}{\partial v_{2}}
\end{array}\right] \text { (B. }
$$

In this case, the inverse form of the Jacobian matrix can be expressed as follows:

$$
\begin{aligned}
& {[\mathbf{J}]^{-1}=} \\
& {\left[\begin{array}{cc}
1.022 v_{1}-0.008 v_{2}+0.01 & -0.008 v_{1} \\
-0.008 v_{2} & 1.016 v_{2}-0.008 v_{1}+0.055
\end{array}\right]_{(\mathrm{B} .3)}^{-1}}
\end{aligned}
$$

The simplification of the previous equation yields the following:

$$
\begin{aligned}
& {[\mathbf{J}]^{-1}=\frac{1}{D}} \\
& {\left[\begin{array}{cc}
500\left(8 v_{1}-1016 v_{2}-55\right) & -4000 v_{1} \\
-4000 v_{2} & -1000\left(511 v_{1}-4 v_{2}+5\right)
\end{array}\right],} \\
& D=4088 v_{1}^{2}+4064 v_{2}^{2}-51917 v_{1} v_{2}-28065 v_{1} \\
& \quad-4860 v_{2}-275 .
\end{aligned}
$$

Assuming the velocities of the previous step as the initial approximation, we can estimate the roots of Eq. (37) as follows:

$$
\{\mathbf{v}\}_{0}=\left\{\begin{array}{l}
3 \\
4
\end{array}\right\} .
$$

Eq. (B.5) may be expressed as:

$$
\stackrel{i=0}{\longrightarrow}\{\mathbf{v}\}_{1}=\{\mathbf{v}\}_{0}-[\mathbf{J}]_{0}^{-1}\{\mathbf{f}\}_{0}
$$

results in:

$$
\begin{aligned}
\{\mathbf{v}\}_{1}= & \left\{\begin{array}{l}
3 \\
4
\end{array}\right\}-\left[\begin{array}{ll}
0.3285 & 0.0019 \\
0.0026 & 0.2442
\end{array}\right]\left\{\begin{array}{l}
0.0360 \\
0.4640
\end{array}\right\} \\
& \rightarrow\{\mathbf{v}\}_{1}=\left\{\begin{array}{l}
2.9873 \\
3.8866
\end{array}\right\} .
\end{aligned}
$$

By inserting the obtained value in the sequence of Eq. (B.1) and continuing calculations until convergence is achieved, the accuracy of the solution can be increased. Note that this quantity, $[\mathbf{J}]^{-1}$, within the Modified Newton Raphson is determined only once in the iteration and is assumed to be constant during the next iterations [60].

\section{Appendix C. Derivation of the exact solution of Example 4.1 by Laplace transform}

Considering Eq. (33):

$$
\left\{\begin{array}{l}
\ddot{x}_{1}+0.22 \dot{x}_{1}-0.16 \dot{x}_{2}+2 x_{1}-x_{2}=0 \\
\ddot{x}_{2}-0.16 \dot{x}_{1}+0.16 \dot{x}_{2}-x_{1}+x_{2}=0
\end{array}\right.
$$

Taking Laplace transform and allowing $F=L\left(x_{1}\right)$ and $G=L\left(x_{2}\right)$, we can get:

$$
\stackrel{L}{\rightarrow}\left\{\begin{array}{c}
s^{2} F-s x_{1(0)}-\dot{x}_{1(0)}+0.22 s F-0.22 x_{1(0)} \\
-0.16 s G+0.16 x_{2(0)}+2 F-G=0 \\
s^{2} G-s x_{2(0)}-\dot{x}_{2(0)}-0.16 s F+0.16 x_{1(0)} \\
\quad+0.16 s G-0.16 x_{2(0)}-F+G=0
\end{array}(\text { C. } 1)\right.
$$

where $s$ is the transformed variable and the zero subscript denotes the initial value at $t=0$.

Imposing the initial conditions and solving this system algebraically for $F$ and $G$, we obtain the following:

$$
\begin{aligned}
& F=\frac{s^{3}+3.38 s^{2}+4.1296 s+7.06}{s^{4}+0.38 s^{3}+3.0096 s^{2}+0.22 s+1} \\
& G=\frac{2 s^{3}+4.76 s^{2}+6.3792 s+11.22}{s^{4}+0.38 s^{3}+3.0096 s^{2}+0.22 s+1}
\end{aligned}
$$


Eq. (C.2) may be written in terms of partial fractions as follows:

$$
\begin{aligned}
F= & \frac{-0.1307-0.106 i}{s-(-0.1733+1.608 i)} \\
& +\frac{-0.1307+0.106 i}{s-(-0.1733-1.608 i)} \\
& +\frac{0.6307-2.1314 i}{s-(-0.0167+0.618 i)} \\
& +\frac{0.6307+2.1314 i}{s-(-0.0167-0.618 i)} \\
G= & \frac{0.0766+0.0718 i}{s-(-0.1733+1.608 i)} \\
& +\frac{0.0766-0.0718 i}{s-(-0.1733-1.608 i)} \\
& +\frac{0.9234-3.4692 i}{s-(-0.0167+0.618 i)} \\
& +\frac{0.9234+3.4692 i}{s-(-0.0167-0.618 i)}
\end{aligned}
$$

Consequently, by taking the inverse transform and simplifying it, displacements of the system $\left(x_{1}, x_{2}\right)$ will be given by:

$$
\stackrel{L^{-1}}{\longrightarrow}\left\{\begin{aligned}
x_{1}= & -0.336 e^{-0.1733 t} \cos (1.608 t+0.681) \\
& +4.445 e^{-0.0167 t} \cos (0.618 t-1.283) \\
x_{2}= & 0.210 e^{-0.1733 t} \cos (1.608 t+0.753) \quad(\text { C. } .4) \\
& +7.180 e^{-0.0167 t} \cos (0.618 t-1.3106)
\end{aligned}\right.
$$

\section{Appendix D. Derivation of the exact solution of Example 4.2 by the Laplace transform}

The following governing equations will be solved by the Laplace transform method:

$$
\left\{\begin{array}{l}
\ddot{x}_{1}+0.2 \dot{x}_{1}-0.1 \dot{x}_{2}+2 x_{1}-x_{2}=\cos t \\
\ddot{x}_{2}-0.1 \dot{x}_{1}+0.2 \dot{x}_{2}-0.1 \dot{x}_{3}-x_{1}+2 x_{2}-x_{3}=\cos 2 t \\
\ddot{x}_{3}-0.1 \dot{x}_{2}+0.1 \dot{x}_{3}-x_{2}+x_{3}=\cos 3 t \quad(48 \mathrm{rep} .)
\end{array}\right.
$$

By $F=L\left(x_{1}\right), G=L\left(x_{2}\right)$, and $H=L\left(x_{3}\right)$ and considering the Laplace transform, we have:

$$
\left\{\begin{aligned}
s^{2} F & -s x_{1(0)}-\dot{x}_{1(0)}+0.2 s F-0.2 x_{1(0)} \\
& -0.1 s G+0.1 x_{2(0)}+2 F-G=\frac{s}{s^{2}+1} \\
s^{2} G & -s x_{2(0)}-\dot{x}_{2(0)}-0.1 s F+0.1 x_{1(0)} \\
& +0.2 s G-0.2 x_{2(0)}-0.1 s H+0.1 x_{3(0)} \\
& \quad-F+2 G-H=\frac{s}{s^{2}+4} \\
s^{2} H & -s x_{3(0)}-\dot{x}_{3(0)}-0.1 s G+0.1 x_{2(0)} s \\
& +0.1 s H-0.1 x_{3(0)}-G+H=\frac{s^{2}+9}{s^{2}}
\end{aligned}\right.
$$

Imposing the zero initial condition and obtaining $F, G$, and $H$, we have:

$$
\left\{\begin{array}{c}
\left(s^{2}+0.2 s+2\right) F+(-0.1 s-1) G+(0) H=\frac{s}{s^{2}+1} \\
(-0.1 s-1) F+\left(s^{2}+0.2 s+2\right) G+(-0.1 s-1) H \\
=\frac{s}{s^{2}+4} \\
(0) F+(-0.1 s-1) G+\left(s^{2}+0.1 s+1\right) H=\frac{s}{s^{2}+9}
\end{array}\right.
$$

Rearranging yields Eq. (D.3) as shown in Box III, where:

$$
\begin{aligned}
A= & 1000 s^{12}+500 s^{11}+19060 s^{10}+8201 s^{9}+125870 s^{8} \\
& +41614 s^{7}+369360 s^{6}+81049 s^{5}+491630 s^{4} \\
& +57936 s^{3}+266080 s^{2}+10800 s+36000 .
\end{aligned}
$$

Eq. (D.4) may be written in terms of partial fractions as follows:

$$
\begin{aligned}
F= & \frac{0.213 s-0.001}{s^{2}+0.02 s+0.198}+\frac{-0.868 s-0.403}{s^{2}+0.155 s+1.555} \\
& +\frac{-0.451 s+0.387}{s^{2}+0.325 s+3.247}+\frac{0.926 s+0.28}{s^{2}+1} \\
& +\frac{0.1830 s-0.546}{s^{2}+4}+\frac{-0.002 s+0.006}{s^{2}+9},
\end{aligned}
$$

$\left\{\begin{array}{l}F=\frac{1000 s^{9}+400 s^{8}+17030 s^{7}+5500 s^{6}+88280 s^{5}+17300 s^{4}+145490 s^{3}+9800 s^{2}+49000 s}{A} \\ G=\frac{1000 s^{9}+500 s^{8}+15050 s^{7}+5800 s^{6}+62430 s^{5}+15300 s^{4}+110620 s^{3}+12400 s^{2}+62000 s}{A} \\ H=\frac{1000 s^{9}+500 s^{8}+10060 s^{7}+4200 s^{6}+40480 s^{5}+17300 s^{4}+12100 s^{3}+13200 s^{2}+66000 s}{A}\end{array}\right.$ 


$$
\begin{aligned}
G= & \frac{0.383 s-0.001}{s^{2}+0.02 s+0.198}+\frac{-0.386 s-0.179}{s^{2}+0.155 s+1.555} \\
& +\frac{0.563 s-0.483}{s^{2}+0.325 s+3.247}+\frac{-0.028 s+0.092}{s^{2}+1} \\
& +\frac{-0.549 s+0.726}{s^{2}+4}+\frac{0.017 s+-0.025}{s^{2}+9}, \\
H= & \frac{0.477 s-0.002}{s^{2}+0.02 s+0.198}+\frac{0.696 s+0.323}{s^{2}+0.155 s+1.555} \\
& +\frac{-0.25 s+0.215}{s^{2}+0.325 s+3.247}+\frac{-0.944 s-0.184}{s^{2}+1} \\
& +\frac{0.148 s-0.335}{s^{2}+4}+\frac{-0.127 s+0.019}{s^{2}+9} .
\end{aligned}
$$

Finally, by taking the inverse transform and simplifying it, the displacements of the system $\left(x_{1}, x_{2}, x_{3}\right)$ will be given by:

$$
\left\{\begin{aligned}
x_{1}= & 0.2131 \exp (-0.01 t) \cos (0.4449 t+0.0330) \\
& -0.9089 \exp (-0.0775 t) \cos (1.2446 t-0.3013) \\
& -0.5188 \exp (-0.1625 t) \cos (1.7946 t+0.5171) \\
& +0.9674 \cos (t-0.2936)+0.3286 \cos (2 t+0.9803) \\
& +0.002 \cos (3 t+3.9267) \\
x_{2}= & 0.3831 \exp (-0.01 t) \cos (0.4449 t+0.0282) \\
& +0.4042 \exp (-0.0775 t) \cos (1.2446 t+2.8406) \\
& +0.6476 \exp (-0.1625 t) \cos (1.7946 t+0.517) \\
& +0.0962 \cos (t+4.4169) \\
& +0.6581 \cos (2 t+3.7258) \\
& +0.0189 \cos (3 t+0.4542) \\
& 0.4772 \exp (-0.01 t) \cos (0.4449 t+0.0318) \\
& +0.7288 \exp (-0.0775 t) \cos (1.2446 t-0.3012) \\
& +0.4097 \exp (-0.1625 t) \cos (1.7946 t+4.0561) \\
& +0.9618 \cos (t+2.9491) \\
& +0.2235 \cos (2 t+0.8471) \\
& +0.1271 \cos (3 t+3.1911)
\end{aligned}\right.
$$

\section{Biographies}

Mohammad Jalili Sadr Abad graduated from Sharif University of Technology with MS degree in
Civil Engineering. In 2018, his PhD in Structural Engineering was completed at Shahid Rajayee University, Lavizan, Tehran, Iran. He is a member of the Iranian Building Engineering Organization as a structural designer. Furthermore, he has teaching experience as a lecturer at the University of Dehkhoda, Ghazvin, and the Azad University of Rudehen. He also worked as a Teacher Assistant at both of Sharif University of Technology and Azad University of Science and Research, Tehran. His primary areas of research interest include nonlinear dynamic analysis, numerical methods, and analysis of dams using FEM.

Mussa Mahmoudi is an Associate Professor at the Department of Civil Engineering at Shahid Rajaee University, Lavizan, Tehran, Iran. Dr. Mahmoudi received his MS and PhD degrees in the Earthquake and Structural Engineering from the Tarbiat Modares University. He wrote three books on structural and earthquake engineering, entitled "philosophy of performance-based seismic design", "column base-plate connection design in steel structures", and "fundamentals of seismic design of structures". Moreover, he has several publications in the following research areas: earthquake engineering; nonlinear analysis, steel structures, and retrofitting.

Earl Dowell is a William Holland Hall Professor of Mechanical Engineering in the Edmund T. Pratt, Jr. School of Engineering at Duke University, Durham NC, USA. Dr. Dowell received his BS degree from the University of Illinois and his SM and ScD degrees from the Massachusetts Institute of Technology. Before coming to Duke as the Dean of the School of Engineering, serving from 1983-1999, he taught at M.I.T. and Princeton. Dr. Dowell research ranges over the topics of aeroelasticity, nonsteady aerodynamics, and nonlinear dynamics. In addition to being the author of over two hundred research articles, Dr. Dowell is the author or co-author of four books, "Aeroelasticity of Plates and Shells", "A Modern Course in Aeroelasticity", "Studies in Nonlinear Aeroelasticity", and "Dynamics of Very High Dimensional Systems". His teaching includes the disciplines of acoustics, aerodynamics, and dynamics. 